\title{
Die Stimme des Volkes. Kommunikations- und Meinungsbildungsprozesse bei lokal- und bundespolitischen Entscheidungen
}

\author{
Metag, Julia
}

\begin{abstract}
Medien Kommunikationswissenschaft bietet ein Forum zur Diskussion medien- und kommunikationsbezogener Fragen sowie für Analysen der Medienentwicklung aus unterschiedlichen Perspektiven und für alle Medien. Medien Kommunikationswissenschaft spiegelt damit die Trends der Forschung und der öffentlichen Diskussion um die Medien wider. Die Zeitschrift hat einen Jahresumfang von ca. 600 Seiten und enthält Aufsätze, Berichte, Dokumente, Diskussionsbeiträge, Rezensionen sowie eine Zeitschriftenlese und eine Literaturübersicht.
\end{abstract}

DOI: https://doi.org/10.5771/1615-634X-2015-1

Posted at the Zurich Open Repository and Archive, University of Zurich ZORA URL: https://doi.org/10.5167/uzh-115296

Journal Article

Published Version

Originally published at:

Metag, Julia (2015). Die Stimme des Volkes. Kommunikations- und Meinungsbildungsprozesse bei lokal- und bundespolitischen Entscheidungen. Medien Kommunikationswissenschaft, 63(1):18-43.

DOI: https://doi.org/10.5771/1615-634X-2015-1 


\section{ResearchGate}

See discussions, stats, and author profiles for this publication at:

http://www.researchgate.net/publication/273081370

\section{Die Stimme des Volkes. \\ Kommunikations- und}

Meinungsbildungsprozesse bei

lokal- und bundespolitischen

Entscheidungen

ARTICLE · JANUARY 2015

DOI: $10.5771 / 1615-634 x-2015-1-18$

READS

68

1 AUTHOR:

Julia Metag

University of Zurich

17 PUBLICATIONS 31 CITATIONS

SEE PROFILE 


\title{
Die Stimme des Volkes
}

\author{
Kommunikations- und Meinungsbildungsprozesse bei lokal- und bundespolitischen \\ Entscheidungen
}

Julia Metag

Direktdemokratische Verfabren werden häufig kontrovers diskutiert. Dabei wird erörtert, ob die Bürger sich in ibrer Meinungsbildung beeinflussen lassen, ausreichend informiert seien und zur Beteiligung angeregt würden. Direkte Demokratie existiert in Deutschland bisher auf substaatlicher, nicht aber auf bundespolitischer Ebene. Der unterschiedliche Einsatz direktdemokratischer Verfabren auf Lokal- und Bundesebene wird letztlich damit begründet, dass Meinungsbildungs- und Kommunikationsprozesse auf lokaler Ebene anders ablaufen als bei bundespolitischen Entscheidungen. Daher untersucht diese Studie, wie sich Medienwirkungen und Rezeptionsprozesse in lokalen und nationalen Öffentlichkeiten unterscheiden. Auf Basis einer Befragung ( $n=1014)$ zu lokalen und nationalen Themen wird analysiert, wie sich Wirkungsprozesse auf die Meinung der Bürger zu den Themen, ibre Kompetenz und Beteiligungsbereitschaft unterscheiden. Es zeigt sich, dass die Meinung der Bürger zu lokal- und bundespolitischen Themen stark prädisponiert ist. Auf lokaler Ebene fübren Mediennutzung und persönliche Gespräche zu einer besseren Kompetenz, wogegen bei nationalen Themen die Medien stärker die Beteiligungsbereitschaft fördern als bei lokalen Themen. Die Befunde werden vor dem Hintergrund direktdemokratischer Verfabren diskutiert.

Schlüsselwörter: Meinungsbildung, Partizipation, Volksentscheid, lokale Kommunikation

\section{Einleitung}

Direktdemokratische Verfahren ermöglichen den Bürgern, selbst politische Entscheidungen zu treffen und stellen daher ein relevantes, wenn auch oftmals kontrovers diskutiertes politisches Element dar. Steht eine direktdemokratische Abstimmung an, wird vor allem über drei Aspekte diskutiert: Wie bilden sich die Bürger eine Meinung über den Abstimmungsgegenstand? Sind sie kompetent genug, darüber direkt selbst abzustimmen? Und werden sie sich an der Abstimmung beteiligen? Das Potenzial zur differenzierten Meinungsbildung wird bei Volksabstimmungen als hoch angesehen, weil die Bürger argumentativ von einer Sachfrage überzeugt werden müssen und es ein großes Spektrum an Akteuren gibt (Marcinkowski \& Marxer, 2011). Da die Bürger politische Entscheidungen direkt beeinflussen, wird von ihnen außerdem eine besonders ausgeprägte Kompetenz bezüglich der zu treffenden Entscheidung gefordert (Kriesi, 2005). Darüber hinaus sollten sich möglichst viele Bürger an einer solchen Abstimmung beteiligen, damit der Entscheid eine hohe Legitimation erfährt. Öffentliche Kommunikation ist für alle drei genannten Aspekte bedeutsam, da sie die Meinungsbildung der Bürger, ihre Kompetenz und Partizipationsbereitschaft beeinflussen kann (Bowler \& Donovan, 1998; Kriesi, 2005).

Mit direkter Demokratie ist hier die Ergänzung des repräsentativ-demokratischen Systems durch Volksentscheide zu verstehen (Schiller \& Mittendorf, 2002). Da das Volk so selbst über politische Sachverhalte entscheiden kann, wird direkte Demokratie häufig als ideales Instrument beteiligungszentrierter Demokratietheorien aufgeführt (Kriesi, 
2005). In Deutschland gibt es direktdemokratische Entscheidungsmöglichkeiten auf Ebene der Kommunen als Bürgerentscheid und auf Landesebene als Volksentscheid. Gleichzeitig entzündet sich an bestimmten politischen Entscheidungen immer wieder eine intensive Debatte um die Frage, ob direktdemokratische Abstimmungen auf Bundesebene in Form von bundesweiten Volksentscheiden eingeführt werden sollen (Kaden, 2010; Schuster, 2011). Die Einwände, die gegen Volksentscheide auf Bundesebene vorgebracht werden, sind vielfach in einer Differenzierung von Lokal- und Bundespolitik begründet. Bundespolitische Probleme werden als zu komplex eingeordnet, als dass die Bürger darüber direkt eine Entscheidung fällen könnten. Wenn die Bürger überhaupt zu einer kompetenten Entscheidung in der Lage seien, dann auf lokaler Ebene, da lokale Themen weniger kompliziert seien (Kost, 2006; Schiller \& Mittendorf, 2002). ${ }^{1}$ Außerdem befürchten Kritiker direktdemokratischer Verfahren eine Beeinflussung der Meinung der Bürger durch Kampagnen in den Medien und anderen Formen öffentlicher Kommunikation. Dies geht so weit, dass von einer möglichen Manipulation der Bürger gesprochen wird (Bowler \& Donovan, 1998). Zudem drohe die Gefahr, dass die Bürger eine Abstimmung nutzen, um ihre Unzufriedenheit mit der Regierung oder einzelnen Parteien auszudrücken (Marcinkowski \& Marxer, 2011). Entsprechend würde das Votum von sachfremden Strategien überlagert. Kommunalpolitik sei dagegen häufig sachbezogener und ideologiefreier als Bundespolitik (Gabriel \& Brettschneider, 1997), sodass die Wahrscheinlichkeit von sachfremden Strategien bei lokalpolitischen Entscheidungen geringer sei. Selbst wenn die Bürger im kommunalen Raum eine „falsche“, also nicht kompetente oder von sachfremden Argumenten geleitete Entscheidung treffen, hätte dies nicht so weitreichende Folgen wie auf Bundesebene (Hartleb \& Jesse, 2005). Zuletzt wird moniert, dass die Beteiligung an direktdemokratischen Abstimmungen zu gering (Kriesi, 2005) und zu selektiv sei, da sich vorrangig die ohnehin politisch interessierten Bürger beteiligten (Hobolt, 2007). Auch wenn Daten zur Wahlbeteiligung das Gegenteil zeigen, wird argumentiert, dass lokalpolitische Entscheidungen die Bürger eher interessieren und sie sich von ihnen betroffen fühlen (Kost, 2006, Vetter, 2011).

Aus Perspektive politischer Kommunikation wird der unterschiedliche Einsatz direktdemokratischer Verfahren auf Lokal- und Bundesebene letztlich damit begründet, dass Meinungsbildungs- und Kommunikationsprozesse auf lokaler Ebene anders ablaufen als bei bundespolitischen Entscheidungen. So wird beispielsweise angenommen, dass die Medien im lokalen Raum im Vergleich zur nationalen Öffentlichkeit weniger Einfluss auf die Meinung der Bürger nehmen, da auf lokaler Ebene ein größerer Einfluss interpersonaler Kommunikation bei politischen Prozessen besteht (Lang, 2003; Tenscher, Seeber \& Hallermayer, 2013). Solche Differenzierungen sind aber bisher wenig theoretisch und empirisch fundiert. Zum einen ist unklar, inwieweit sich Medienwirkungs- und Rezeptionsprozesse in lokalen und nationalen Öffentlichkeiten unterscheiden. Zum anderen wurde die Wirkung dieser Prozesse auf die Meinung zu politischen Themen, die Kompetenz der Rezipienten und ihre Beteiligungsbereitschaft bisher nicht systematisch vergleichend untersucht. Daher fragt diese Studie: Unterscheiden sich die Kommunikations- und Meinungsbildungsprozesse der Bürger bei lokal-und bundespolitischen Entscheidungen? Welche Implikationen für die Debatte um bundesweite Volks-

1 Es gibt in der Politikwissenschaft auch Debatten darüber, welche Themen sich für Sachabstimmungen eignen, da verschiedene Themen von unterschiedlicher Tragweite sind. So wird z.B. diskutiert, ob Bürger direkt über den Bundeshaushalt oder außenpolitische Themen entscheiden sollten (Hartleb \& Jesse, 2005). Da in Deutschland die Debatte über Lokal- und Bundesebene bei direktdemokratischen Verfahren mehrheitlich unabhängig von konkreten Themen geführt wird, ist es aber zunächst relevant, diese Unterschiede themenübergreifend zu prüfen. 
entscheide lassen sich daraus folgern? Der Beitrag vergleicht die Kommunikationswirkungen auf die Meinung, Kompetenz und Partizipationsabsichten der Rezipienten systematisch.

\section{Forschungsstand}

Bei einer direktdemokratischen Abstimmung ist die Meinung der Bürger zur Sachfrage zentral. Aus demokratietheoretischer Sicht kann die Meinungsbildung dabei sehr differenziert verlaufen, da die Bürger argumentativ überzeugt werden müssen (Marcinkowski \& Marxer, 2011). Außerdem soll die Meinungsbildung auf Basis eines hinreichenden Wissensbestandes über ein Thema erfolgen (Schmitt-Beck, 2000). Damit ist Kompetenz die zweite demokratietheoretisch wichtige Variable. Partizipation an der Abstimmung steht darüber hinaus bei dieser beteiligungszentrierten Demokratieform im Zentrum.

Die Forschung zur politischen Meinungsbildung zeigt, dass ähnliche Determinanten die Meinung der Bürger, ihre Kompetenz bei einem politischen Thema sowie ihre Beteiligungsbereitschaft beeinflussen. Bei allen drei Konstrukten sind Wirkungen durch mediale und interpersonale Kommunikation möglich, da diese Informationen zum Abstimmungsgegenstand bereitstellen (Kriesi, 2005; Marcinkowski \& Marxer, 2011). Die politische Involvierung und Betroffenheit der Rezipienten stellen zudem relevante Einflussfaktoren dar. Die Meinung zu einer politischen Entscheidung ist darüber hinaus von den Prädispositionen der Rezipienten geprägt (Zaller, 1992). Außerdem werden die Kompetenz der Bürger sowie ihre Meinung von der Art, wie sie Informationen verarbeiten, mitbestimmt (Eagly \& Chaiken, 1993). Die Beteiligungsbereitschaft ist wiederum ebenfalls von der Meinung und Kompetenz der Rezipienten abhängig. Die Einflüsse der Faktoren auf die Meinung zu politischen Themen, die Kompetenz und Partizipationsabsichten sind weiter unten in Abbildung 1 veranschaulicht.

\subsection{Determinanten der Meinungsbildung}

\subsubsection{Medienwirkungen auf die Meinung}

Studien zur Wirkung von Massenkommunikation auf das Wählerverhalten und bei direktdemokratischen Abstimmungen zeigen, dass die Massenmedien das Potenzial besitzen, die Meinung der Bürger zu beeinflussen (de Vreese, 2007; de Vreese \& Semetko, 2004; Kriesi, 2005; Schmitt-Beck, 2000: 321-330). Allerdings sind tatsächliche Meinungsänderungen der Rezipienten eher weniger wahrscheinlich, da durch die Mediennutzung vorrangig die schon vorhandenen Einstellungen der Rezipienten verstärkt werden (Iyengar \& Simon, 2000).

Lokale Kommunikationsräume und Medieninhalte zeichnen sich durch einige Besonderheiten aus, wie zum Beispiel das gemeinsame Wissen der Rezipienten über den Lokalraum oder die lokale Identität (Reinemann, Fawzi \& Röder, 2012; Tenscher et al., 2013). Die Kommunikation der politischen Akteure ist im Lokalen auch geringer professionalisiert (Koch, Baden, Klötzer \& Müller, 2013; Metag \& Marcinkowski, 2012). Generell ist der lokale Kontext, wie die lokale Medienlandschaft, publizistische Vielfalt und politische Kommunikationskultur, zentral für die Ausgestaltung lokaler politischer Kommunikation (Baugut \& Reinemann, 2013; van Deth \& Tausendpfund, 2013).

In Studien zu lokalen Themen, wie zum Beispiel kommunalen oder regionalen Wahlkämpfen, werden Wirkungen der Medien auf die politische Meinung der Bürger gefunden (Bachl \& Vögele, 2013; Neller, 1999; Scheufele, Shanahan \& Kim, 2002). Unterschiede lassen sich bezüglich der Art der Informationsquelle ausmachen - bei lokalen 
Themen wird überwiegend die lokale Tageszeitung genutzt und bei nationalen Themen das Fernsehen (Hasebrink \& Schmidt, 2012). Da bisher kein systematischer Vergleich von Medienwirkungen zwischen Lokal- und Bundesebene vorliegt, bleibt die Frage offen, ob Medienwirkungen auf die Meinung in der nationalen Öffentlichkeit stärker sind und von anderen Medientypen ausgehen als in lokalen Öffentlichkeiten.

Auch interpersonale Kommunikation hat einen Einfluss auf die politische Meinung der Bürger (Kim, Wyatt \& Katz, 1999; Sommer, 2010) - auch bei Volksentscheiden (Marcinkowski \& Marxer, 2011). Interpersonale Kommunikation wird bei der Information über lokalpolitische Themen häufiger von den Rezipienten genutzt als bei der Information über nationale Themen (Hasebrink \& Schmidt, 2012; Tenscher, 2013). Daher lässt sich vermuten, dass der Einfluss auf die Meinung durch persönliche Gespräche bei lokalpolitischen Themen stärker ist als bei bundespolitischen Themen.

\subsubsection{Wirkungen von Prädispositionen auf die Meinung}

Studien zu Volksentscheiden machen deutlich, dass politische Prädispositionen wie Ideologie, Wertvorstellungen und Parteiidentifikation relevant für die Meinungsbildung sind, da sie die Zuwendung zu und Akzeptanz von Informationen beeinflussen (Kriesi, 2005, 2012; Zaller, 1992). Die Wahlforschung zeigt, dass die Parteiidentifikation bei bundespolitischen Entscheidungen meist wirkungsmächtiger für die Wahlentscheidung ist als bei kommunalen Wahlen (Marcinkowski, 2001). Ideologische Einstellungen und Wertorientierungen sind dagegen relativ stabil und auf kommunaler und bundespolitischer Ebene gleichermaßen relevant (Roßteuscher \& Scherer, 2013; Winter, 1997). Darüber hinaus können themenspezifische Prädispositionen gerade bei direktdemokratischen Verfahren die Meinung beeinflussen (Kriesi, 2012), da solche Entscheidungen sachbezogen sind und es weniger um politische Parteien und Kandidaten geht. Damit sind Einstellungen der Rezipienten gemeint, die sie $\mathrm{zu}$ einem bestimmten politischen Themenbereich haben (Kriesi, 2012), zum Beispiel beim Thema Asylrecht das Ausmaß der Fremdenfeindlichkeit einer Person. Da themenspezifische Prädispositionen von der konkreten Sachfrage abhängen, dürfte sich bei ihnen kein Unterschied in der Stärke des Einflusses in Abhängigkeit von der föderalen Ebene zeigen.

\subsubsection{Wirkungen von Involvierung auf die Meinung}

Mit politischer Involvierung sind das politische Interesse einer Person und ihre kognitiven Fähigkeiten gemeint. Bei Personen mit einer starken Involvierung sind Meinungsänderungen durch politische Kommunikation weniger wahrscheinlich, da sie starke Voreinstellungen besitzen (Zaller, 1992). Politisch gering involvierte Bürger sind dagegen leichter zu beeinflussen, kommen mit politischen Informationen aber aufgrund ihres geringen Interesses seltener in Kontakt. Daher geht Zaller (1992) davon aus, dass Personen mit mittlerem Involvement am ehesten anfällig für Persuasionsbotschaften sind.

Frühere Studien argumentieren, dass Bürger auf kommunaler Ebene stärker involviert sind, da lokalpolitische Angelegenheiten sie eher direkt betreffen und leichter verständlich sind als bundespolitische Themen (Becker \& Whitney, 1980; Palmgreen \& Clarke, 1977). Auch wenn aktuelle Daten zur Wahlbeteiligung und zum lokalpolitischen Interesse nicht auf ein stark höheres Interesse an lokalen Themen hindeuten (van Deth \& Tausendpfund, 2013), geben in einer deutschen Repräsentativstudie 46 Prozent der Befragten an, sehr stark an Ereignissen aus dem Ort, in dem sie leben, interessiert zu sein. Im Gegensatz dazu sind 30 Prozent der Befragten sehr stark an Ereignissen aus Deutschland interessiert (Oehmichen \& Schröter, 2011). Auf lokaler Ebene sind das politische 
Interesse und Wissen der Bürger, wenn auch nur leicht, stärker ausgeprägt als auf nationaler Ebene (Mende, Oehmichen \& Schröter, 2012; Shaker, 2009). Daher wird zwar angenommen, dass sich die politische Involvierung bei lokalen und nationalen Entscheidungen unterscheidet, es lassen sich aber im Hinblick auf Zaller (1992) keine differenzierten Annahmen darüber treffen, wie stark dieser Unterschied ist. Deshalb ist keine eindeutige Aussage über die Kommunikationswirkung möglich, da Personen bei lokalpolitischen Entscheidungen beispielsweise nur mittelstark involviert sein könnten.

\subsubsection{Wirkungen von Betroffenheit auf die Meinung}

Rezipienten können sich von lokal- und bundespolitischen Entscheidungen unterschiedlich stark betroffen fühlen. Häufig wird angenommen, dass Bürger direkter von lokalen Entscheidungen betroffen seien (Paletz, Reichert \& McIntyre, 1971; Vetter, 2011). Bei direktdemokratischen Verfahren wird das Problem genannt, die Bürger würden hauptsächlich nach ihrer eigenen Betroffenheit entscheiden (vgl. Bowler \& Donovan, 1998; Verhulst \& Nijeboer, 2007), gleichzeitig zeigt sich aber, dass persönliche Betroffenheit die Nutzung von Informationsquellen zum Abstimmungsgegenstand positiv verstärkt (vgl. Marcinkowski \& Marxer, 2011). Es erscheint jedoch wenig sinnvoll, politische Entscheidungen nach ihrer Betroffenheit einzuordnen, ohne die Perspektive des Rezipienten mit einzubeziehen (Marcinkowski \& Marxer, 2011). Entsprechend lassen sich lokal- und bundespolitische Entscheidungen nicht von vornherein bezüglich der Betroffenheit, die sie auslösen, klassifizieren, und es bleibt zu klären, ob Betroffenheit bei lokalen oder nationalen Themen stärker ist.

\subsubsection{Wirkungen der Informationsverarbeitung auf die Meinung}

Studien zu Volksentscheiden beschäftigen sich oftmals damit, ob alle Bürger ausreichend über die Sachfrage informiert sind (Hobolt, 2007). Dabei wird häufig auf unterschiedliche Informationsverarbeitungswege verwiesen (Eagly \& Chaiken, 1993). Systematische Informationsverarbeitung setzt eine starke Involvierung voraus und führt zu einer stabileren Meinung (Marcinkowski \& Marxer, 2011). Heuristische Verarbeitung tritt dagegen bei geringerer Involvierung auf, trotzdem können Rezipienten durch die Orientierung an Heuristiken zu ähnlich kompetenten Meinungen über einen Abstimmungsgegenstand gelangen (Lupia, 1994). Im Zusammenhang mit politischen Entscheidungen sind die Parteiheuristik, also die Orientierung an der Position einer Partei zu einem Thema, sowie das Vertrauen in Politik von Bedeutung (Hobolt, 2007; Kriesi, 2005). Je nachdem, ob die Involvierung der Rezipienten bei lokalen oder nationalen Themen höher ist, sollte eine systematische Informationsverarbeitung bei politischen Entscheidungen auf der jeweiligen föderalen Ebene stärker sein.

\subsection{Determinanten für die politische Kompetenz}

\subsubsection{Medienwirkungen auf die politische Kompetenz}

Politische Kompetenz umfasst das Wissen und Interesse, das die Bürger zu einem spezifischen Thema haben (Eveland, Hayes, Shah \& Kwak, 2005). Durch die Nutzung von Massenmedien und interpersonaler Kommunikation können Rezipienten ihr politisches Wissen und Interesse verbessern (Maier, 2009; McLeod, Scheufele \& Moy, 1999). Untersuchungen zu EU-Referenden und nationalen Abstimmungen zeigen, dass Mediennutzung und Gespräche das Wissen über einen Abstimmungsgegenstand verbessern 
können (de Vreese \& Semetko, 2004; Marcinkowski \& Marxer, 2011). Das Gleiche gilt für Medienwirkungen bei lokal- und bei bundespolitischen Entscheidungen (Metag, 2013; Moy, McCluskey, Kelley \& Spratt, 2004). Daher bleibt die Frage offen, ob Medienwirkungen auf die Sachkompetenz bei lokal- und bundespolitischen Entscheidungen unterschiedlich stark ausfallen. Da interpersonale Kommunikation für die Information über lokale Themen wichtiger ist, wird davon ausgegangen, dass ihre Wirkung auf die Kompetenz in lokalen Öffentlichkeiten stärker ist als in nationalen Öffentlichkeiten.

\subsubsection{Wirkungen von Involvierung auf die politische Kompetenz}

Die Kompetenz der Bürger, die generell politisch stärker involviert sind, ist zumeist auch bei einer speziellen Sachfrage höher, weil sie sich häufiger und intensiver informieren (Eveland, 2001). Bei Volksentscheidungen in der Schweiz und in Liechtenstein wurde deutlich, dass das generelle politische Interesse der Rezipienten die Informationssuche und die Wissenserweiterung über die Sachfrage positiv verstärkt (Bonfadelli \& Friemel, 2012; Marcinkowski \& Marxer, 2011). Für die vorliegende Fragestellung bedeutet dies: Wenn sich die Bürger generell zum Beispiel mehr für Lokal- als für Bundespolitik interessieren, ist es auch wahrscheinlicher, dass sie bei einer spezifischen lokalpolitischen Entscheidung die Medien und persönliche Gespräche intensiver nutzen als bei einer bundespolitischen Entscheidung - und umgekehrt.

\subsubsection{Wirkungen von Betroffenheit auf die politische Kompetenz}

Die Betroffenheit durch einen Abstimmungsgegenstand beeinflusst, ob die Meinungsbildung darüber systematisch oder heuristisch vollzogen wird, und wirkt sich damit indirekt auf die Kompetenz aus. Je betroffener man sich fühlt, desto häufiger beruht die Entscheidung der Bürger auf einem systematischen Meinungsbildungsprozess (Marcinkowski \& Marxer, 2011) und desto mehr werden Medien und interpersonale Kommunikation genutzt und so das Wissen der Rezipienten verbessert (Hefner, 2012). Da sich Personen nicht a priori von lokalen Entscheidungen stärker betroffen fühlen als von nationalen Entscheidungen, ist unklar, ob es Unterschiede in der Effektstärke von Betroffenheit bei lokal- und bundespolitischen Entscheidungen gibt.

\subsubsection{Wirkungen der Informationsverarbeitung auf die politische Kompetenz}

Die Art der Informationsverarbeitung beeinflusst die Kompetenz, da systematische Verarbeitung in einem größeren Wissen über ein Thema resultiert als heuristische Verarbeitung (Marcinkowski \& Marxer, 2011). Sollten die Bürger Informationen über lokalpolitische Entscheidungen eher systematisch verarbeiten als Informationen über nationale Entscheidungen (Metag, 2013), dann sollte der Einfluss von systematischer Informationsverarbeitung auf die Kompetenz auf lokaler Ebene stärker sein als auf Bundesebene.

\subsection{Determinanten der Partizipationsbereitschaft}

\subsubsection{Medienwirkungen auf Partizipationsbereitschaft}

Durch Informationen aus den Massenmedien und persönlichen Gesprächen werden Themen fokussiert, bei denen Handlungsbedarf besteht, und so die politische Beteiligung gefördert (Scheufele, Nisbet, Brossard \& Nisbet, 2004). Die Beteiligung an Volks- 
entscheiden kann ebenfalls durch direkten Kontakt mit der medialen Kampagne und indirekte Kampagneneffekte, mediatisiert durch politische Gespräche, verstärkt werden (Hobolt, 2007; Schuck \& de Vreese, 2009). Positive Kommunikationswirkungen auf die politische Beteiligung finden sich auch auf lokaler Ebene (Filla \& Johnson, 2010; Nicodemus, 2004), die Frage nach Unterschieden in den Medienwirkungen zur nationalen Ebene ist aber offen. Hinsichtlich persönlicher Gespräche sollte der positive Einfluss auf die politische Partizipationsbereitschaft bei lokalen Themen größer sein als bei nationalen Themen.

\subsubsection{Wirkungen von Involvierung, Kompetenz und Meinung auf Partizipationsbereitschaft}

Rezipienten, die politisch interessiert und kompetent sind, beteiligen sich eher an politischen Prozessen (Verba, Schlozman \& Brady, 1995) und auch an einem Referendum (de Vreese \& Semetko, 2004). Gleichzeitig nutzen involvierte Bürger Informationsquellen häufiger, was wiederum das Mobilisierungspotenzial dieser Quellen erhöht. Analog zur allgemeinen politischen Involvierung wirkt sich auch die themenspezifische Kompetenz, die von dem jeweiligen politischen Thema abhängt, förderlich auf die politische Partizipation aus (Kriesi, 2005, 2007). Darüber hinaus kann es von der Meinung zu einem Thema abhängig sein, ob sich ein Bürger politisch beteiligt. Dies wird bei direktdemokratischen Verfahren deutlich, wenn die Stimmbürger, die gegen den Vorschlag sind, sich erst gar nicht an der Abstimmung beteiligen, damit diese das notwendige Quorum nicht erreicht (Mehr Demokratie Nordrhein-Westfalen, o. J.).

\subsubsection{Wirkungen von Betroffenheit auf die Partizipationsbereitschaft}

Fühlt sich eine Person von einer politischen Entscheidung betroffen, kann sie politisch mobilisiert werden, auch wenn die Person sonst eher gering politisch involviert ist (Bowler \& Donovan, 1998). Direktdemokratische Sachfragen, mit denen die Bürger vertraut sind und von denen sie sich betroffen fühlen, führen zu einer höheren Beteiligung (Kriesi, 2007). Es bleibt empirisch zu prüfen, ob sich die Stärke der Wirkung von Betroffenheit auf politische Partizipationsabsichten auf den beiden föderalen Ebenen unterscheidet.

Die Modelle (Abb. 1) zu den Wirkungen auf die politische Meinung, Kompetenz und Partizipationsbereitschaft werden vergleichend empirisch für lokale und bundespolitische Entscheidungen überprüft, um Implikationen für direktdemokratische Abstimmungen ableiten zu können.

\section{Methode}

\subsection{Datenerbebung}

Im November 2011 wurde eine standardisierte, telefonische Befragung durchgeführt, in der jeder Teilnehmer entweder zu einer bundes- oder einer lokalpolitischen Entscheidung befragt wurde. Eine Befragung ist das geeignete Instrument zur Datenerhebung, da sie die Möglichkeit bietet, Informationen über die individuelle Rezeption von lokalund bundespolitischen Entscheidungen durch die Selbstbeschreibung der Befragten zu erhalten. Um grundsätzliche Aussagen über lokale bzw. nationale Entscheidungen machen zu können, wurde zu je drei verschiedenen Themen befragt, damit Unterschiede auf die föderale Ebene zurückgeführt werden können und nicht auf themenspezifische 


\section{Abbildung 1: Theoretische Wirkungsmodelle}

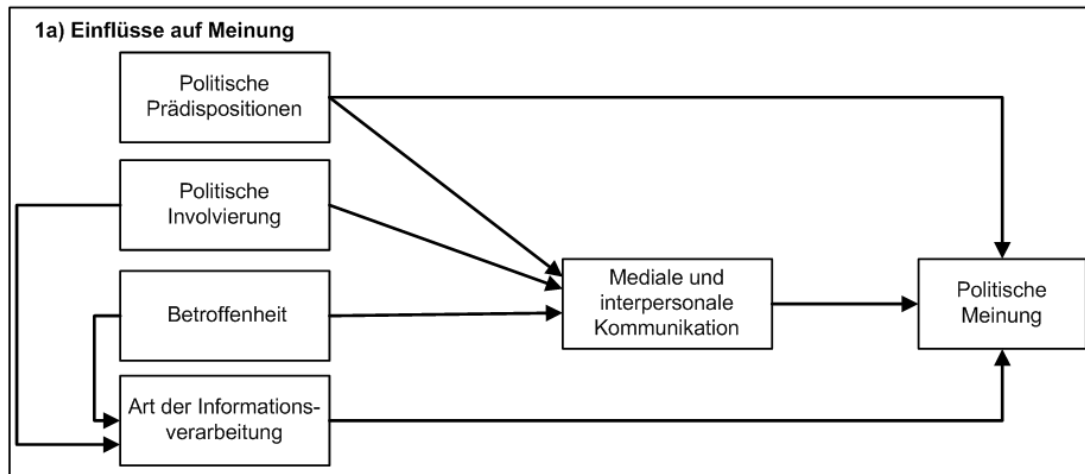

1b) Einflüsse auf Kompetenz

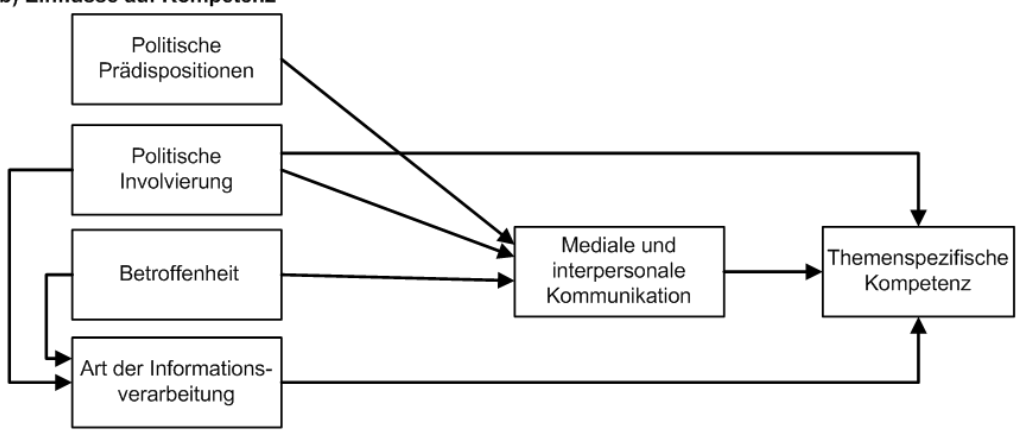

1c) Einflüsse auf Partizipationsbereitschaft

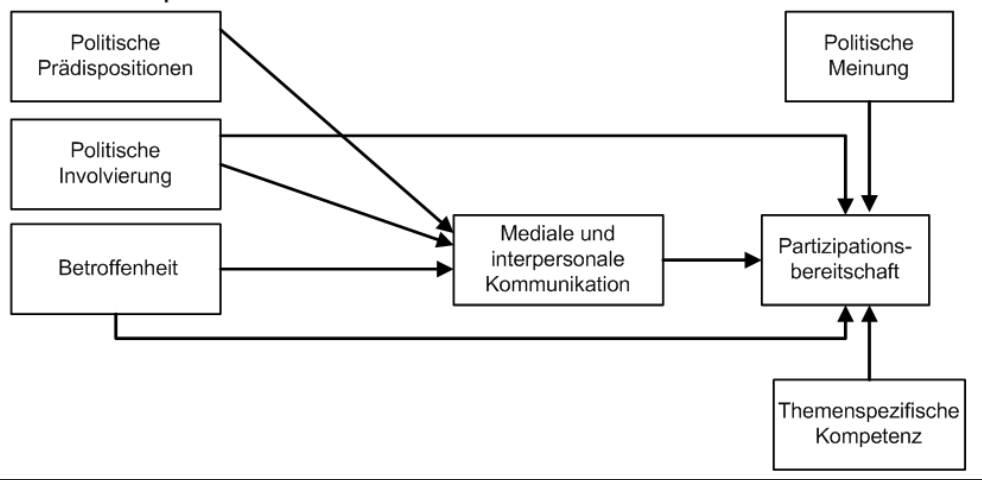

Aspekte. Die drei Entscheidungen wurden jeweils aus dem gleichen Politikfeld gewählt, um ihre Vergleichbarkeit zu gewährleisten. Darüber hinaus war wichtig, dass es sich bei den lokalen Themen um Themen handelt, bei denen es prinzipiell möglich wäre, dass ein Bürgerentscheid stattfindet. Allerdings sollte keine konkrete Abstimmung anstehen, da dies die Vergleichbarkeit zu den bundespolitischen Entscheidungen einschränken würde. 
Da es in Deutschland derzeit nicht möglich ist, die Meinungsbildung vor Volksabstimmungen auf Bundesebene zu untersuchen, ist die Aussagekraft der Studie beschränkt, da die größere öffentliche Aufmerksamkeit während einer direktdemokratischen Kampagne nicht erfasst werden kann. Der hier gewählte methodische Ansatz ermöglicht aber Aussagen über generelle Unterschiede in Meinungsbildungsprozessen zwischen lokalen und nationalen Öffentlichkeiten, die dann bei tatsächlichen Kampagnen überprüft werden können. Außerdem lassen Studien zu Sachabstimmungen in anderen Ländern eine Einordnung im Hinblick darauf zu, ob die hier gefundenen Zusammenhänge auch auf tatsächliche Abstimmungen übertragbar sind.

Ausgewählt wurden aus dem Politikfeld „Energie“ der Bau eines Windparks in Vaterstetten als lokale Entscheidung, die zum Erhebungszeitpunkt gerade vor Ort heiß diskutiert wurde, und der Atomausstieg Deutschlands als nationale Entscheidung. Für das Politikfeld „Verkehr“ wurden der geplante Bau einer Umgehungsstraße in Limburg a. d. Lahn, der schon über mehrere Jahre umstritten ist, und die ebenso kontroverse Diskussion um die PKW-Maut auf nationaler Ebene als Themen bestimmt. Aus dem Politikfeld „Finanzen“ wurden die Debatte um die Planung des Musikzentrums in Bochum als lokale Entscheidung und der Euro-Rettungsschirm als nationales Thema ausgewählt. Bei beiden Themen steht der Umgang mit Steuergeldern im Vordergrund, da das Musikzentrum als Steuerverschwendung kritisiert wurde, weil es im Umkreis schon ähnliche Einrichtungen gibt.

$\mathrm{Zu}$ den drei bundespolitischen Entscheidungen wurden bundesweit 510 Personen und zu den drei lokalpolitischen Entscheidungen 504 Personen befragt. Die Teilstichproben teilen sich gleichmäßig auf die drei lokalen und die drei nationalen Entscheidungen auf, sodass pro Entscheidung eine Stichprobe von rund 170 Personen gezogen wurde. Es wurden demnach sechs Teilbefragungen zu jeweils einem Thema durchgeführt. Durch die parallel gehaltene Befragung handelt es sich hier um ein Ex-post-factoDesign, bei dem in einem Datenerhebungsprozess alle abhängigen und unabhängigen Variablen gemessen werden (Schnell, Hill \& Esser, 2008). Hinsichtlich der Kategorien Alter und Geschlecht sind die Stichproben repräsentativ für die jeweilige Grundgesamtheit. Das durchschnittliche Alter aller Befragten lag bei 52 Jahren (SD=17) und es wurden fast genauso viele Frauen (49 \%, n=493) wie Männer (51 \%, n=521) befragt. Befragte mit Fachoberschulreife oder Abitur $(45 \%, n=552)$ sind dagegen etwas überrepräsentiert.

\subsection{Operationalisierung und Datenanalyse}

Die theoretischen Modelle (Abb. 1) werden mittels Pfadanalyse getestet und die Variablen für die Pfadmodelle mehrschrittig ausgewählt. Auf Basis von bivariaten Korrelationen zwischen den abhängigen und unabhängigen Variablen wurden die Variablen gewählt, die die stärksten Korrelationen zeigten. Wenn es keine signifikanten Korrelationen gab, wurde trotzdem für jedes Konstrukt (z. B. Prädispositionen) mindestens eine Variable einbezogen, da indirekte Effekte möglich sind und so das Konstrukt im Modell zumindest erfasst wird. Außerdem wurde die Fallzahl der Variablen bei der Auswahl berücksichtigt. Tabelle 1 stellt die Operationalisierung der Variablen dar, die in den Pfadmodellen Verwendung finden.

Die Meinung zu den politischen Entscheidungen, die Kompetenz der Befragten und ihre Partizipationsbereitschaft stellen die abhängigen Variablen dar. Die Meinung der Befragten wurde darüber erhoben, ob sie für oder gegen die politische Entscheidung waren. Das Konstrukt Kompetenz wurde operationalisiert als ein Index aus dem Interesse und Wissen der Rezipienten über die spezifische Entscheidung. Dadurch, dass nicht 
tatsächlich ein direktdemokratischer Entscheid anstand, konnte nur die potenzielle Partizipationsbereitschaft erhoben werden.

\section{Tabelle 1: Überblick über Variablen}

\begin{tabular}{l|l}
\hline Konstrukte & Operationalisierung \\
\hline Abhängige Variablen \\
\hline Meinung & $\begin{array}{l}\text { „Mal ganz generell gesprochen - sind Sie für oder gegen XY (z. B. den Atom- } \\
\text { ausstieg)?“; } 1=\text { voll und ganz dagegen }-5=\text { voll und ganz dafür } \\
\text { Lokal: } \mathrm{M}=3.6, \mathrm{SD}=1.5 ; \text { National: } \mathrm{M}=3.48, \mathrm{SD}=1.48\end{array}$ \\
\hline $\begin{array}{l}\text { Themenspezi- } \\
\text { fische Kompe- }\end{array}$ & $\begin{array}{l}\text { Index aus Interesse („Wie stark interessieren Sie sich für das Thema XY?“ } \\
\text { 1=überhaupt nicht }-5=\text { sehr stark) und drei Wissensfragen (zwei Fragen zum } \\
\text { tenz }\end{array}$ \\
$\begin{array}{l}\text { politischen Wissen über die Entscheidung; eine Frage zum allgemeinen Wissen } \\
\text { über Lokal-/Bundespolitik) }\end{array}$ \\
\begin{tabular}{l} 
Lokal: $\mathrm{M}=6.60, \mathrm{SD}=2.12 ;$ National: $\mathrm{M}=7.72, \mathrm{SD}=2.20$ \\
\hline Partizipations- \\
bereitschaft
\end{tabular} & $\begin{array}{l}\text { „Würden Sie sich an einer Abstimmung zum Thema XY beteiligen?“ 1=be- } \\
\text { stimmt nicht }-4=\text { bestimmt }\end{array}$ \\
\hline
\end{tabular}

\section{Unabhängige Variablen}

Kommunikati- $\quad$ „Wie häufig informieren Sie sich über das Thema XY in den folgenden Inforonsnutzung mationsquellen (lokale Tageszeitung, überregionale Tageszeitung, Fernsehen, Radio, Internet, persönliche Gespräche)?“; 1=nie - 4=sehr häufig Lokal: Mediennutzung: $\mathrm{M}=1.94, \mathrm{SD}=.82$, Interpersonale Kommunikation: $\mathrm{M}=2.46, \mathrm{SD}=0.94$

National: Mediennutzung: $\mathrm{M}=2.46, \mathrm{SD}=.94$, Interpersonale Kommunikation: $\mathrm{M}=2.34, \mathrm{SD}=0.87$

\begin{tabular}{l|l}
\hline Themenspezi- & „Ich nenne Ihnen nun fünf politische Themenbereiche und Sie sagen mir bitte
\end{tabular} fische Prädis- $\quad$ jeweils, für wie wichtig Sie diese halten.“; $1=$ überhaupt nicht wichtig $-5=$ sehr positionen wichtig

Faktorenanalyse ${ }^{3}$ Beispiel: Beim Thema Euro-Rettungsschirm laden die Variablen Wirtschafts-, EU- und Außenpolitik auf einen Faktor (Cronbachs alpha=. 70; Faktorladungen zwischen .61 und .75; $\mathrm{KMO}=.67$; erklärte Varianz durch Eigenwerte: $62,79 \%$ ).

\begin{tabular}{l|l}
\hline Betroffenheit & „Wie stark fühlen Sie sich selbst von Thema XY betroffen?“; $1=$ überhaupt nicht
\end{tabular} $-5=$ sehr stark

Lokal: $\mathrm{M}=3.42 ; \mathrm{SD}=1.06 ;$ National: $\mathrm{M}=3.72 ; \mathrm{SD}=.99$

2 Beispiel: „Wie viele Windräder sollen im Ebersberger Forst gebaut werden?“, „Bis zu welchem Jahr will der Landkreis Ebersberg unabhängig von fossilen und endlichen Energieträgern werden?", „Wer ist zurzeit Bürgermeister in Vaterstetten?“. Da es sich teilweise um Schätzungen (z. B. von Kosten, Jahreszahlen) handelte, wurde ein Punktesystem entwickelt: Zwei Punkte für vollständig korrekte Antworten, einen Punkt für ungefähr richtige Antworten, keinen Punkt für falsche Antworten und die Antwort „weiß nicht“. Als ungefähr richtige Antworten wurden Antworten gewertet, die in einem gewissen Toleranzbereich lagen und bei Zahlenwerten über die Standardabweichung bestimmt wurden (z. B. bei der Frage nach der Anzahl der Windräder des Windparks in Vaterstetten wurden als ungefähr richtige Antworten gewertet: 5 und 7 [richtige Antwort: 6]). Insgesamt konnten maximal 6 Punkte erreicht werden. Die Antworten auf die Wissensfragen und die Interessensfrage wurden zu einem Index, der von 1 bis 11 reicht, aufsummiert.

3 Alle Faktorenanalysen in dieser Studie wurden mit Hauptachsenanalyse und Oblimin-Rotation durchgeführt und es wurde vorgegeben, dass alle Items auf einen Faktor laden sollen. 


\begin{tabular}{|c|c|}
\hline Involvierung & $\begin{array}{l}\text { „Wie stark interessieren Sie sich für Bundespolitik/ Lokalpolitik?"; } 1=\text { über- } \\
\text { haupt nicht }-5=\text { sehr stark } \\
\text { Lokal: } \mathrm{M}=3.42 ; \mathrm{SD}=1.06 ; \text { National: } \mathrm{M}=3.72 ; \mathrm{SD}=.99\end{array}$ \\
\hline $\begin{array}{l}\text { Systematische } \\
\text { Informations- } \\
\text { verarbeitung }\end{array}$ & $\begin{array}{l}\text { „Je mehr Informationen ich zum Thema XY finde, desto besser“, „Es ist mir } \\
\text { wichtig, alle Argumente zum Thema XY im Detail zu kennen“; } 1=\text { stimme über- } \\
\text { haupt nicht zu }-5=\text { stimme voll und ganz zu } \\
\text { Faktorenanalyse: Cronbachs alpha=.77, KMO=.52, erklärte Varianz durch Ei- } \\
\text { genwerte: } 73,09 \% \text {, Faktorladungen für beide Items }=.79\end{array}$ \\
\hline $\begin{array}{l}\text { Vertrauen in } \\
\text { Responsivität }\end{array}$ & $\begin{array}{l}\text { „Auf Bundesebene/ In meiner Stadt hat meine Stimme bei politischen Ent- } \\
\text { scheidungen Gewicht.“; } 1=\text { stimme überhaupt nicht zu }-5=\text { stimme voll und } \\
\text { ganz zu } \\
\text { Faktorenanalyse: Cronbachs alpha=.70; KMO=.50; erklärte Varianz durch Ei- } \\
\text { genwerte: } 76,87 \% \text {, Faktorladungen für beide Items=.73 }\end{array}$ \\
\hline Parteiheuristik & $\begin{array}{l}\text { Antworten der Befragten auf die Frage „Bitte geben Sie an, ob die jeweilige } \\
\text { Partei Ihrer Meinung nach eher für das Thema XY ist, eher gegen das Thema } \\
\text { XY ist oder ihm neutral gegenübersteht“ wurden daraufhin kodiert, ob sie der } \\
\text { tatsächlichen Position der jeweiligen Partei entsprechen. } \\
\text { Lokal: } 41 \% \text { richtige Einschätzungen }(n=425) \text {; National: } 47 \% \text { richtige Ein- } \\
\text { schätzungen }(n=463)\end{array}$ \\
\hline \multicolumn{2}{|c|}{ Kontrollvariablen } \\
\hline $\begin{array}{l}\text { Demokratiezu- } \\
\text { friedenheit }\end{array}$ & $\begin{array}{l}\text { „Wie zufrieden sind Sie mit der Demokratie, so wie sie in Deutschland be- } \\
\text { steht?"; } 1=\text { überhaupt nicht zufrieden }-5=\text { sehr zufrieden } \\
\text { Lokal: } M=3.46 ; \mathrm{SD}=1.00 ; \text { National: } \mathrm{M}=3.34 ; \mathrm{SD}=1.03\end{array}$ \\
\hline $\begin{array}{l}\text { Erfahrung mit } \\
\text { direkter Demo- } \\
\text { kratie }\end{array}$ & $\begin{array}{l}\text { „Haben Sie schon einmal mitbekommen, dass es an Ihrem derzeitigen oder frü- } \\
\text { heren Wohnort einen Bürger- oder Volksentscheid gab?" } \\
\text { Lokal: } 1=\mathrm{ja}(61 \%), 0=\text { nein }(39 \%), \mathrm{n}=483 \text {; National: } 1=\mathrm{ja}(47 \%), 0=\text { nein } \\
(53 \%), \mathrm{n}=483\end{array}$ \\
\hline $\begin{array}{l}\text { Wunsch nach } \\
\text { einer Abstim- } \\
\text { mung }\end{array}$ & $\begin{array}{l}\text { „Wie stark würden Sie sich wünschen, über XY direkt in einem Bürgerentscheid } \\
\text { abstimmen zu können?“; } 1=\text { überhaupt nicht }-5=\text { sehr stark } \\
\text { Lokal: } M=3.61 ; \mathrm{SD}=1.43 \text {; National: } \mathrm{M}=3.50 ; \mathrm{SD}=1.50\end{array}$ \\
\hline $\begin{array}{l}\text { allgemeine po- } \\
\text { litische Partizi- } \\
\text { pation }\end{array}$ & $\begin{array}{l}\text { „Sind Sie politisch aktiv, also z. B. Mitglied in einer Partei, einem Verband, einer } \\
\text { Gewerkschaft etc.?“ } \\
\text { Lokal: } 1=j a(15 \%), 0=\text { nein }(85 \%), n=483 \text {; National: } 1=j a(15 \%), 0=\text { nein } \\
(85 \%), n=489\end{array}$ \\
\hline
\end{tabular}

Die theoretischen Modelle spezifizieren die unabbängigen Variablen der Studie. Die Kommunikationsprozesse der Rezipienten wurden über die Häufigkeit der spezifischen Nutzung verschiedener Massenmedien und persönlicher Gespräche gemessen. Durch die Häufigkeit der Mediennutzung kann nicht direkt die Wirkung der Inhalte der Berichterstattung untersucht werden, da keine Daten über die Konsonanz oder Vielfalt dieser Inhalte vorliegen. Um sich den Medieninhalten zu nähern, wurden die Befragten nach ihrer Wahrnehmung gefragt, ob sich die Medien zu einer politischen Entscheidung positionieren („Wie würden Sie die Berichterstattung in den Medien insgesamt zum Thema $\mathrm{XY}$ einschätzen? Eher dafür, eher dagegen, neutral, teils/teils?“). Bei beiden föderalen Ebenen gaben jeweils 60 Prozent der Befragten an, dass die Berichterstattung überwiegend neutral oder ausgewogen war. Keine signifikanten Unterschiede gab es darüber hinaus in der wahrgenommenen Informationsqualität der Berichterstattung („Wie gut fühlen Sie sich von den Medien über Thema XY informiert? " $1=$ gar nicht - 5=sehr gut; lokal: $\mathrm{M}=3.21$; $\mathrm{SD}=.93$; national: $\mathrm{M}=3.16 ; \mathrm{SD}=.92$ ). Entsprechend kann davon ausge- 
gangen werden, dass es zumindest in der Wahrnehmung der Rezipienten wenig Unterschiede in der Medienberichterstattung über lokale und nationale Themen gab.

Als themenspezifische Prädispositionen wird die persönliche Wichtigkeit von verschiedenen politischen Themenbereichen abgefragt. Dabei sind die Themenbereiche übergreifender als die konkrete Sachfrage, aber berühren diese. Weisen Rezipienten eine starke Prädisposition im Hinblick auf einen solchen generellen Themenbereich auf, sollte diese für die konkrete Sachfrage relevant werden. So wird beispielsweise der Aufgabenbereich Energiepolitik beim Thema Atomausstieg abgefragt. ${ }^{4}$ Betroffenheit wird als wahrgenommene Betroffenheit der Rezipienten abgefragt. Da politische Involvierung ein stabiles, themenunabhängiges Konstrukt ist, wird das allgemeine Interesse der Befragten an Bundes- respektive Lokalpolitik als Operationalisierung verwendet. So wird trotzdem zwischen den föderalen Ebenen unterschieden. Dies ist sinnvoll, da die Wahlbeteiligung deutlich macht, dass die politische Involvierung der Bürger auf den föderalen Ebenen unterschiedlich ausgeprägt sein kann. Häufig wird das Bildungsniveau der Befragten für die Operationalisierung der kognitiven Komponente von Involvierung genutzt (Marcinkowski \& Marxer, 2011). Da das Bildungsniveau unabhängig von der föderalen Ebene ist und hier nicht mit der Meinung zu den politischen Entscheidungen korreliert, beschränkt sich die Operationalisierung auf die motivationale Komponente politischer Involvierung.

Systematische Informationsverarbeitung wurde über zwei Items erhoben, die sich für ihre Messung etabliert haben (Schemer, Wirth \& Matthes, 2008). Als heuristische Informationsverarbeitungsstrategie wurde einerseits das Vertranen in die Responsivität von Lokal-und Bundespolitik verwendet. Wenn Rezipienten politische Institutionen als responsiv wahrnehmen, ist es wahrscheinlicher, dass sie sich auch bei ihrer Meinungsbildung an ihnen orientieren, da sie sich eher auf deren Einschätzung einer Sachfrage verlassen. Die Parteiheuristik misst andererseits, inwieweit die Position einer Partei zur Orientierung genutzt werden kann. Für Einflüsse auf die Meinung zu einem Thema ist die Wahrnehmung der Position der Parteien zentral, unabhängig davon, ob diese Wahrnehmung sachlich richtig ist. Diese Heuristik hat sich für das finale Analysemodell zur Meinung aber nicht als relevant dargestellt. Parteiheuristiken können auch mit der Kompetenz der Befragten zusammenhängen. Wer weiß, wie Parteien zu einer politischen Entscheidung stehen, sollte auch besser über die Aspekte dieser Entscheidung Bescheid wissen. Daher wird gemessen, ob die Befragten die Positionen einer Partei zu einem Thema richtig einschätzen können. Im Unterschied zur themenspezifischen Kompetenz geht es hier aber nicht um Detailwissen zur Sachfrage, sondern um das Wissen um die Position einer Partei zu der Entscheidung.

Abgesehen vom Alter, Geschlecht und der Bildung der Befragten wurden für die Wirkungen auf die Partizipationsbereitschaft die Demokratiezufriedenheit der Bürger, ihre Erfahrung mit direkter Demokratie, ihre allgemeine politische Partizipation und ihr Wunsch nach einer Abstimmung als Kontrollvariablen erhoben. Es ist wahrscheinlich, dass Personen, die sich eine Abstimmung wünschen, sich dann auch daran beteiligen. Gleichzeitig ist denkbar, dass sich jemand nicht unbedingt eine Abstimmung wünscht, wenn sie dann aber ansteht, sich trotzdem daran beteiligen will, um gerade die Durchsetzung der Sachfrage zu verhindern. Um die reine Partizipationsbereitschaft unabhängig vom Wunsch nach der Abstimmung zu erklären, wird dieser Abstimmungswunsch als Kontrollvariable verwendet.

4 Es wurden als Prädispositionen auch Parteineigung und politische Ideologie erhoben. Beide Variablen gehen aber nicht in die finalen Analysemodelle ein. 
Die Pfadmodelle für lokale und bundespolitische Entscheidungen wurden mittels Maximum-Likelihood-Schätzung separat geschätzt, da teilweise verschiedene Variablen verwendet wurden, sodass ein Multipler Gruppenvergleich nicht möglich war. ${ }^{5}$ Die Modelle wurden zunächst auf Basis der theoretischen Modelle geschätzt und danach schrittweise angepasst, um optimale Modelle zu erzielen, die die Daten am besten abbilden. Die Anpassungen wurden auf Basis der Modifikations-Indizes in AMOS durchgeführt. Tabelle 2 (Anhang) zeigt eine Korrelationsmatrix der in den Modellen verwendeten Variablen.

\section{Ergebnisse}

Der Test der verschiedenen Modelle demonstriert Unterschiede hinsichtlich zweier Vergleichsdimensionen. Zum einen unterscheiden sich die Einflussfaktoren, die bei den jeweiligen abhängigen Variablen wirksam werden. Zum anderen werden Unterschiede zwischen den Einflussprozessen bei lokalen und bei nationalen Themen deutlich.

\subsection{Wirkungen auf die Meinung}

Die Modelle für die Meinung zu lokal- und bundespolitischen Entscheidungen (Abb. 2) zeigen eine gute Anpassung an die Daten (lokal: $\mathrm{p}=.585, \chi 2(20)=18.039$, RMSEA $=.00$, $\mathrm{CFI}=1.00$; national: $\mathrm{p}=.068 ; \chi 2(15)=23.86 ; \mathrm{RMSEA}=.038 ; \mathrm{CFI}=.981)$. Für lokalpolitische Entscheidungen wurde die Nutzung der lokalen Tageszeitung (lokal: $\mathrm{M}=3.18 ; \mathrm{SD}=.83$ ) und für bundespolitische Entscheidungen die Fernsehnutzung (national: $M=2.80 ; \mathrm{SD}=$. 82) als Variablen für Mediennutzung verwendet, da diese Medien für die Information über die jeweiligen Themen von den Rezipienten am häufigsten genutzt wurden.

Das Pfadmodell für lokalpolitische Entscheidungen (Abb. 2a) zeigt keine signifikanten Wirkungen von Tageszeitungsnutzung und interpersonaler Kommunikation auf die Meinung zur lokalen Entscheidung. Die Meinung wird dagegen von den themenspezifischen Prädispositionen der Rezipienten beeinflusst sowie von systematischer Informationsverarbeitung. Je wichtiger den Rezipienten mit dem Thema verwandte Politikfelder sind und je intensiver sie sich mit Informationen über das lokale Thema auseinandersetzen, umso eher befürworten sie die lokalpolitische Entscheidung. Darüber hinaus zeigt sich, dass ein hohes Vertrauen in die Responsivität der kommunalen Politik eher zu einer Zustimmung zur politischen Entscheidung führt. Fühlen sich die Bürger außerdem von der lokalen Entscheidung betroffen, sind sie eher dafür, da sie vielleicht von dem neuen Musikzentrum oder dem vom Windpark erzeugten Ökostrom profitieren können.

Eine Mediationsanalyse weist auf zwei signifikante indirekte Effekte hin. ${ }^{6}$ Persönliche Betroffenheit wirkt sich vermittelt durch die Nutzung interpersonaler Kommuni-

5 Multiple Gruppenvergleiche, bei denen Gruppe 1 die Befragten zu lokalpolitischen Themen und Gruppe 2 die Befragten zu bundespolitischen Themen wären, würden einen strengeren Test der Unterschiede zwischen lokalen und nationalen Themen bieten. Dieses Vorgehen wurde getestet, aber die Fit-Werte der Modelle verschlechterten sich signifikant, wenn in beiden Gruppen exakt die gleichen Variablen modelliert wurden. Da das Ziel dieser Studie die Untersuchung der unterschiedlichen Wirkzusammenhänge zwischen den beiden föderalen Ebenen ist, wurde sich für die Analyse von zwei verschiedenen Modellen entschieden, die jeweils gut die Daten abbilden.

6 Indirekte Effekte: Betroffenheit $\rightarrow$ interpersonale Kommunikation $\rightarrow$ Meinung $=-.024 *(a=.16$, $\mathrm{Sa}=.033 ; \mathrm{b}=-.153, \mathrm{Sb}=.083)$; Nutzung lokale Tageszeitung $\rightarrow$ interpersonale Kommunikation $\rightarrow$ Meinung $=-.018 *(\mathrm{a}=.120, \mathrm{Sa}=.057 ; \mathrm{b}=-.153, \mathrm{Sb}=.083)$. 
kation signifikant auf die Meinung zu einer lokalen Entscheidung aus. Auch die Nutzung der lokalen Tageszeitung übt eine Wirkung auf die Meinung aus, wenn Rezipienten über

\section{Abbildung 2: Wirkungen auf Meinung ${ }^{7}$}

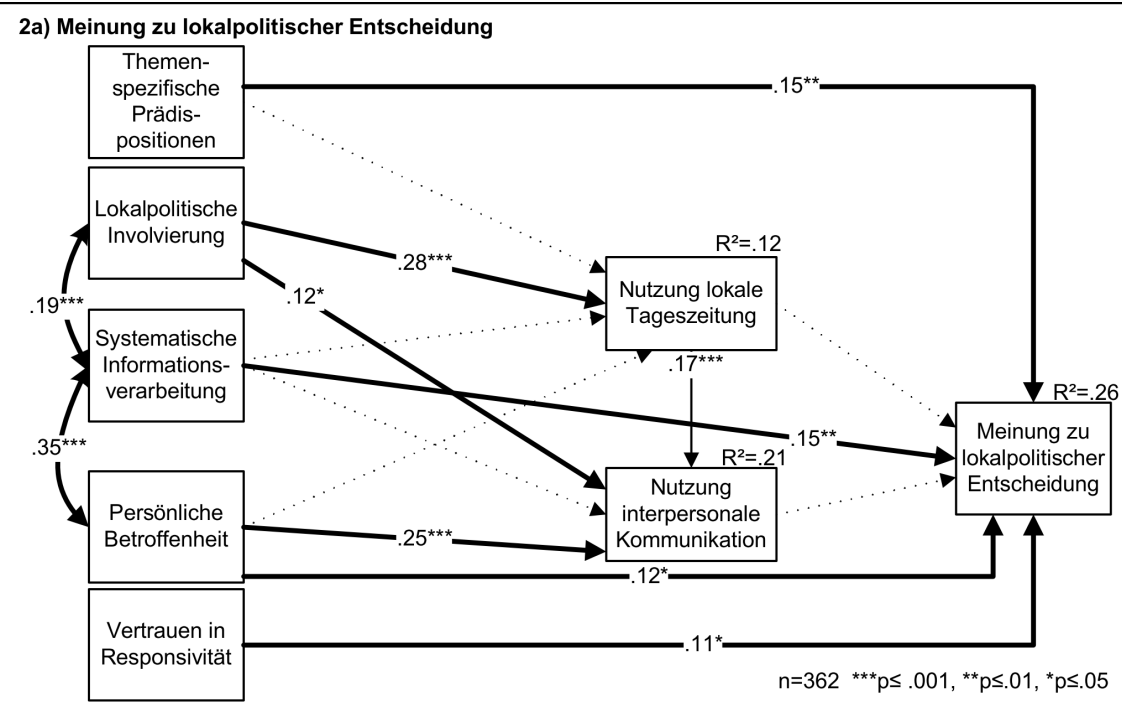

2b) Meinung zu bundespolitischer Entscheidung

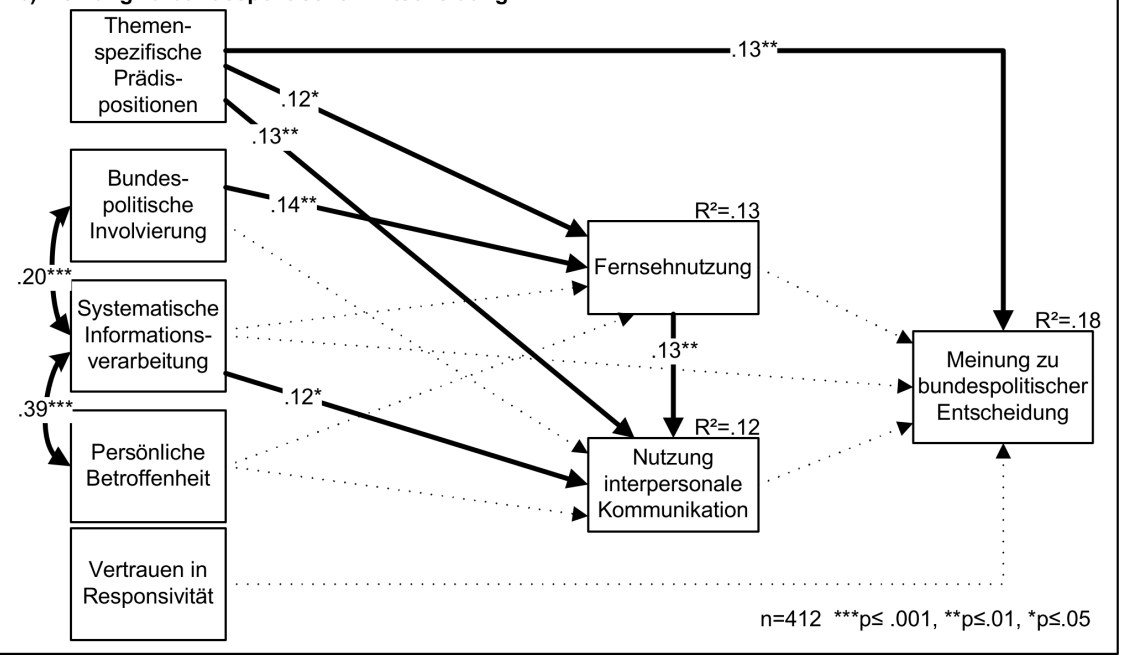

7 In den Abb. 2-4 stellen gestrichelte Pfeile nicht-signifikante Pfade dar, durchgezogene Pfeile signifikante Pfade mit standardisierten Regressionskoeffizienten. Um die Abbildungen übersichtlich zu halten, sind die Koeffizienten der nicht-signifikanten Pfade sowie die Kontrollvariablen und Korrelationen zwischen exogenen Variablen, die nicht in den theoretischen Modellen vermutet wurden, nicht dargestellt. 
das, was sie in der lokalen Tageszeitung lesen, sprechen. Interpersonale Kommunikation hat also doch eine Wirkung auf die Meinung der Rezipienten, wenn eine lokalpolitische Entscheidung eine starke Betroffenheit auslöst oder die Bürger regelmäßig Tageszeitung lesen. Beide Effekte sind negativ, persönliche Gespräche über die lokalen Themen führen also eher zu einer Meinung gegen den politischen Entscheidungsvorschlag. Vielleicht werden in den Gesprächen stärker kritische Argumente angesprochen, z. B. Probleme wie Lärm und Schattenschlag durch einen Windpark.

Auch bei nationalen Entscheidungen (Abb. 2b) haben mediale und interpersonale Kommunikation keine Wirkung auf die Meinung und es gibt ebenso einen direkten Effekt der themenspezifischen Prädispositionen. Systematische Informationsverarbeitung hat keinen direkten Effekt auf die Meinung, und auch das Vertrauen in politische Responsivität nicht. Im Gegensatz zum Modell zur Meinung zu lokalen Entscheidungen gibt es hier keine signifikanten indirekten Effekte der unabhängigen Variablen auf die Meinung. Die Meinung der Rezipienten zu bundespolitischen Entscheidungen basiert also vor allem auf den spezifischen Prädispositionen, die die Befragten dem jeweiligen Thema gegenüber haben. Ist ihnen zum Beispiel Umweltpolitik generell wichtig, befürworten sie auch die bundespolitische Entscheidung zum Atomausstieg.

\subsection{Wirkungen auf die politische Kompetenz}

Bei den Modellen für die Wirkungszusammenhänge auf die Kompetenz (Abb. 3; lokal: $\mathrm{p}=.094 ; \chi 2(64)=79.328$; RMSEA=.026; $\mathrm{CFI}=.982$; national: $\mathrm{p}=.053 ; \chi 2(47)=63.698 ; \mathrm{RM}-$ SEA=.029; CFI=.981) wurden die Tageszeitungs-, Internet- und Fernsehnutzung als Mediennutzungsvariablen integriert.

Wie vermutet haben Tageszeitungsnutzung und persönliche Gespräche einen positiven Effekt auf die lokalpolitische Kompetenz (Abb. 3a). Internetnutzung hat im multivariaten Pfadmodell keinen signifikanten Effekt mehr. Dies legt die Vermutung nahe, dass die Internetnutzung auf lokaler Ebene ein Proxy für Involvierung darstellt und es vor allem politisch involvierte Personen sind, die das Internet zur Information über lokalpolitische Ereignisse nutzen. Konform mit den theoretischen Überlegungen ist, dass es einen positiven Effekt von politischer Involvierung und systematischer Informationsverarbeitung auf die themenspezifische Kompetenz gibt. Je involvierter die Rezipienten sind und je intensiver sie sich mit Informationen über die lokalpolitische Entscheidung auseinandersetzen, desto kompetenter sind sie. Die Vertrauens- und Parteiheuristik sind im multivariaten Modell bei lokalpolitischen Entscheidungen nicht mehr bedeutsam. In lokalen Öffentlichkeiten sind Parteien weniger relevant für Informationen über eine politische Entscheidung, vermutlich, da parteipolitische Ausrichtungen in der Kommunalpolitik eine weniger zentrale Rolle spielen. Allerdings zeigt das Pfadmodell, dass, wer sich als persönlich betroffen von der Entscheidung wahrnimmt, auch kompetenter ist, denn betroffene Rezipienten sind interessierter an der lokalen Entscheidung.

Betroffenheit und Involvierung beeinflussen die lokalpolitische Kompetenz der Befragten auch indirekt vermittelt über die Nutzung von persönlichen Gesprächen. Indem Rezipienten, die sich betroffen fühlen oder ohnehin politisch involviert sind, sich häufiger Diskussionen über das jeweilige Thema zuwenden, ist ihre Kompetenz diesbezüglich besser als bei Personen, die weniger betroffen und interessiert sind. Je involvierter Rezipienten sind und je systematischer sie sich mit Informationen auseinandersetzen, desto eher nutzen sie außerdem die lokale Tageszeitung und sind dadurch kompetenter. Persönliche Gespräche bewirken außerdem die Weitergabe von Informationen aus der lokalen Tageszeitung und können so die themenspezifische Kompetenz der Rezipienten 
Abbildung 3: Wirkungen auf Kompetenz

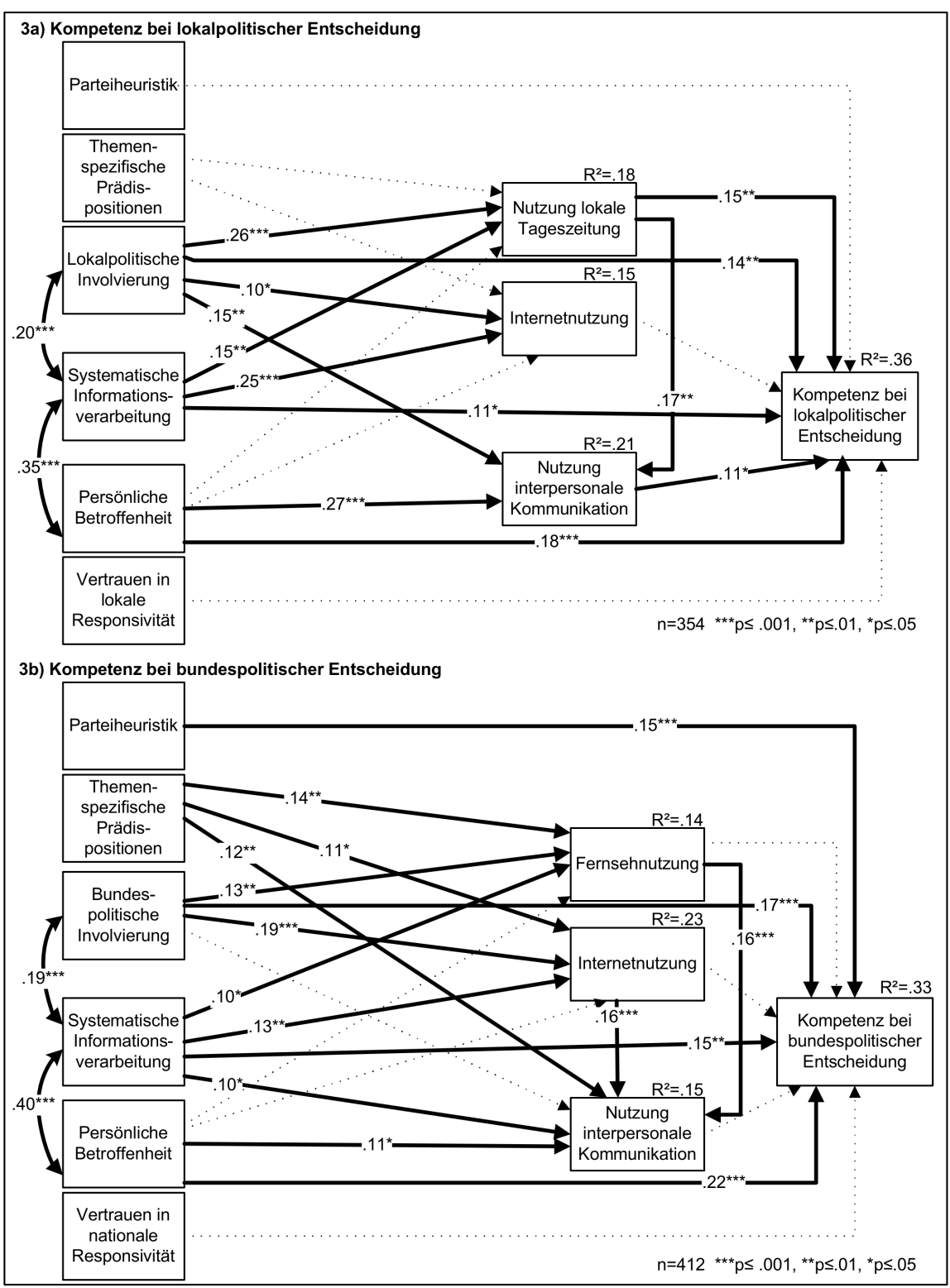


steigern. Wenn die Befragten sich häufig zu der lokalen Entscheidung in der Tageszeitung informieren, führt dies zu häufigeren Diskussionen über das Thema. ${ }^{8}$

Im Gegensatz zum Pfadmodell zur Kompetenz bei lokalen Entscheidungen zeigen die drei Kommunikationskanäle bei nationalen Entscheidungen (Abb. 3b) keine signifikanten Wirkungen. Ebenso wie bei lokalpolitischen Entscheidungen haben Involvierung, systematische Informationsverarbeitung und Betroffenheit einen positiven Effekt auf die Kompetenz der Befragten, unabhängig davon, welche Informationen sie aus Medien und persönlichen Gesprächen rezipieren. Bei bundespolitischen Entscheidungen stützt sich die Sachkompetenz der Bürger eher auf die Parteiheuristik. Wenn die Bürger über die Positionen der Parteien Bescheid wissen, können sie eine kompetente Entscheidung fällen. Entsprechende Informationen über die Parteipositionen erhalten sie wahrscheinlich vorrangig aus den Massenmedien, sodass der Einfluss der Parteiheuristik hier vermutlich auch als Medieneinfluss interpretiert werden kann.

\subsection{Wirkungen auf die Partizipationsbereitschaft}

Der Index der Mediennutzung zeigt bei lokalen und bei nationalen Entscheidungen die stärksten Zusammenhänge mit der Beteiligung und wird daher in den Modellen für die Partizipationsbereitschaft verwendet (Abb. 4; lokal: $\mathrm{p}=.063 ; \chi 2(27)=38.993$; RMSEA=. 036; CFI=.963; national: $\mathrm{p}=.409 ; \chi 2(21)=21.844$; RMSEA=.010; CFI=.998). Ebenso werden die Meinung der Bürger ${ }^{9}$ und ihre Kompetenz zum Thema, die in 4.1 und 4.2 die abhängigen Variablen waren, in die Modelle integriert, da beide mit der Beteiligungsabsicht zusammenhängen.

Interpersonale Kommunikation und Mediennutzung haben keinen signifikanten Effekt auf die Beteiligungsbereitschaft bei lokalen Themen (Abb. 4a). Wichtig für die potenzielle Partizipation an einer lokalen Abstimmung ist dagegen, dass überhaupt der Wunsch nach dieser Abstimmung besteht und die Rezipienten eine gewisse Kompetenz aufweisen. Auch Betroffenheit, politische Involvierung, eine ausgeprägte Meinung zum Thema sowie allgemeine politische Partizipation und die Erfahrung mit direkter Demokratie zeigen keine signifikanten Wirkungen.

Bei bundespolitischen Entscheidungen (Abb. 4b) wirkt sich dagegen Mediennutzung positiv auf die Partizipationsbereitschaft aus. Rezipienten scheinen durch die Mediennutzung Informationen zu erhalten, die sie zur Beteiligung an einem potenziellen bundesweiten Volksentscheid benötigen oder die sie mobilisieren.

Korrespondierend $\mathrm{zu}$ den lokalen Entscheidungen beeinflussen auch der Wunsch nach einer Abstimmung und die themenspezifische Kompetenz die Partizipationsbereitschaft auf nationaler Ebene positiv. Auf beiden föderalen Ebenen zeigt sich gleicher-

8 Indirekte Effekte: Involvierung $\rightarrow$ Tageszeitungsnutzung $\rightarrow$ Kompetenz $=.078 *(a=.213, \mathrm{Sa}=$. 043; b=.366, Sb=.121); Systematische Informationsverarbeitung $\rightarrow$ Tageszeitungsnutzung $\rightarrow$ Kompetenz $=.048 *(\mathrm{a}=.132, \mathrm{Sa}=.045 ; \mathrm{b}=.366, \mathrm{Sb}=.121)$; Tageszeitungsnutzung $\rightarrow$ interpersonale Kommunikation $\rightarrow$ Kompetenz $=.047 *(a=.19, \mathrm{Sa}=.058 ; \mathrm{b}=.245, \mathrm{Sb}=.105)$; Betroffenheit $\rightarrow$ interpersonale Kommunikation $\rightarrow$ Kompetenz $=.044 *(\mathrm{a}=.179, \mathrm{Sa}=.032 ; \mathrm{b}=.245, \mathrm{Sb}=.105)$; Involvierung $\rightarrow$ interpersonale Kommunikation $\rightarrow$ Kompetenz $=.036 *(a=.147, \mathrm{Sa}=.049 ; \mathrm{b}=.245, \mathrm{Sb}=$. 105).

9 Die Beziehung zwischen Meinung und Partizipationsbereitschaft ist nicht-linear, da die Beteiligungsbereitschaft jeweils auf den Polen der Meinungsskala am stärksten ausgeprägt ist. Daher bildet eine neue Variable die extreme Meinung zu einer politischen Entscheidung ab. Die Variable ist dichotom mit $1=$ extreme Meinung und $0=$ moderate Meinung kodiert (Die Ausprägungen 1 und 5 wurden als $1=$ extreme Meinung kodiert, die Ausprägungen 2-4 als $0=$ moderate Meinung). 


\section{Abbildung 4: Wirkungen auf Partizipationsbereitschaft}

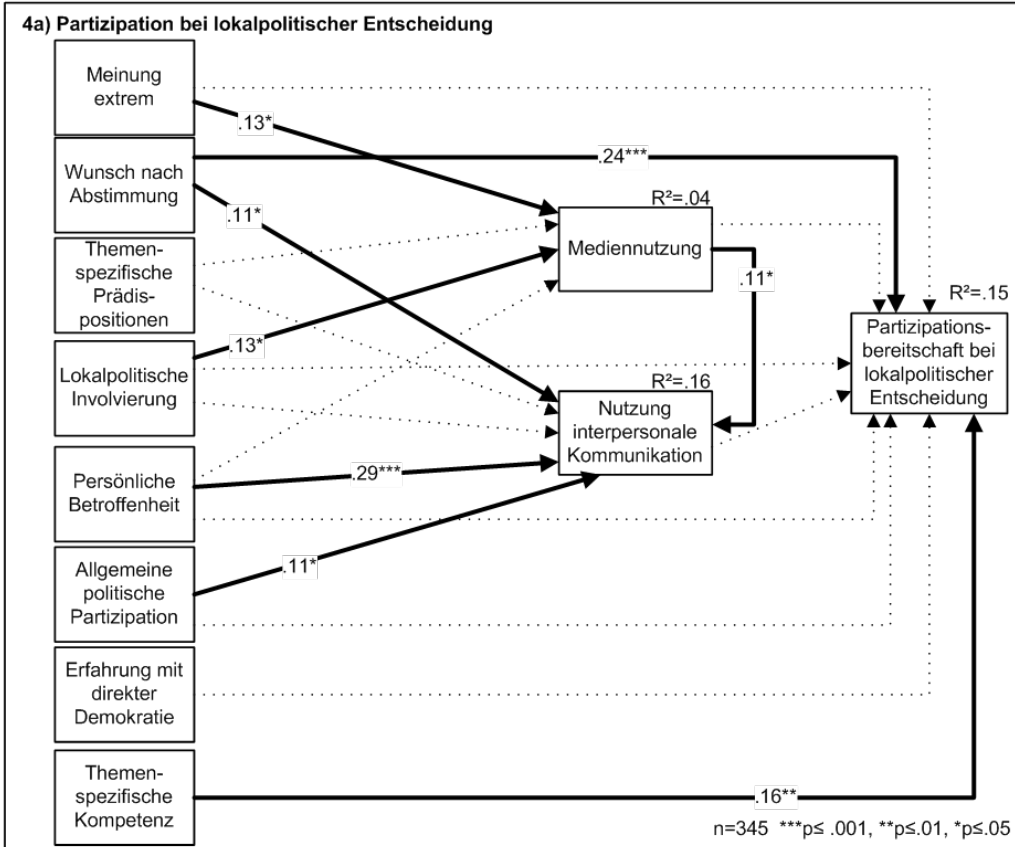

4b) Partizipation bei bundespolitischer Entscheidung

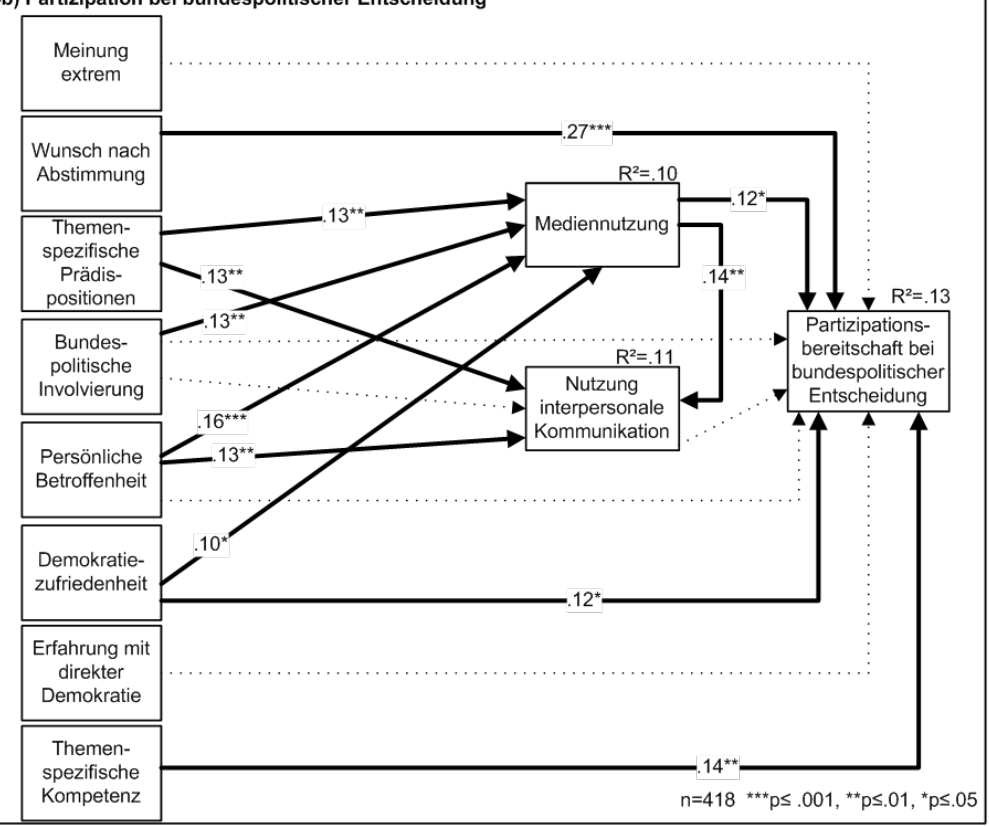


maßen die Tendenz, dass es vor allem die ohnehin politisch interessierten und zur Sachfrage kompetenten Bürger sind, die sich an einem direktdemokratischen Entscheid beteiligen würden. Bei bundespolitischen Entscheidungen wird darüber hinaus deutlich, dass Bürger, die insgesamt mit der Demokratie in Deutschland zufrieden sind, sich eher an einem Volksentscheid beteiligen würden als politikverdrossene und unzufriedene Bürger.

Durch den signifikanten Effekt von Mediennutzung auf die Partizipationsbereitschaft bei nationalen Themen gibt es einige indirekte Wirkungen. ${ }^{10}$ Eine starke Betroffenheit, ausgeprägte themenspezifische Prädispositionen, eine hohe Involvierung und Demokratiezufriedenheit führen dazu, dass die Rezipienten häufiger die Medien zur Information über bundespolitische Entscheidungen nutzen und sich daraufhin eher an einem Volksentscheid beteiligen würden.

\section{Diskussion}

Aus den Befunden zu den Wirkungen auf die Meinung, Kompetenz und Partizipationsbereitschaft bei lokal- und bundespolitischen Entscheidungen lässt sich schließen, dass die Prozesse politischer Kommunikation mehrheitlich nicht die Differenzierung zwischen den beiden föderalen Ebenen, die bei direkter Demokratie in Deutschland vorgenommen wird, unterstützen. Zwar verlaufen Kommunikations- und Rezeptionsprozesse bei lokal- und bundespolitischen Entscheidungen unterschiedlich. Diese Differenzen gestalten sich aber überwiegend nicht so, dass deshalb direktdemokratische Abstimmungen auf Bundesebene abgelehnt werden sollten, wenn man sie von einem normativen Standpunkt aus generell als wünschenswert ansieht.

In Bezug auf Medienwirkungen unterscheidet sich die Meinungsbildung in lokalen und nationalen Öffentlichkeiten wenig, da die Bürger ihre Meinung kaum aufgrund ihrer Mediennutzung ändern. Da es nicht möglich war, Kommunikations- und Meinungsbildungsprozesse im Vorfeld tatsächlich stattfindender Volksentscheide zu untersuchen, kann dies dazu führen, dass der Einfluss der Medien unterschätzt wird. Während eines Volksentscheids ist die Medienberichterstattung intensiver, und die verschiedenen Akteure versuchen mit ihren Kampagnen, die Rezipienten in ihrer Entscheidung zu beeinflussen. Die vorliegende Studie setzt gewissermaßen davor an und reflektiert die $\mathrm{Zu}$ sammenhänge von Kommunikation, relevanten Drittvariablen und den abhängigen Variablen auf lokaler und nationaler Ebene. Aber auch wenn bei Volksentscheiden die Medienberichterstattung sicherlich intensiver ist, zeigen Arbeiten zu direktdemokratischen Kampagnen, dass Meinungsänderungen auch dabei eher unwahrscheinlich sind (Marcinkowski \& Marxer, 2011). Der Einfluss schon bestehender Prädispositionen ist bedeutsamer. Diese Studie zeigt, dass dies auf beiden föderalen Ebenen der Fall ist. Der Einfluss von themenspezifischen Prädispositionen legt eine Meinungsbildung auf Basis von Sachargumenten nahe, was demokratietheoretisch normativ erwünscht ist. Wenn überhaupt, sind Meinungsänderungen durch persönliche Gespräche - bei stark betroffenen Rezipienten und durch Tageszeitungsnutzung angeregte Gespräche - möglich.

Auch wenn bei Volksentscheiden nur mit ja oder nein abgestimmt werden kann und generelle Einstellungen für die Meinung zu einer spezifischen Sachfrage bedeutsam sind,

10 Indirekte Effekte: themenspezifische Prädispositionen $\rightarrow$ Mediennutzung $\rightarrow$ Partizipation $=$. $014 \%(\mathrm{a}=.124, \mathrm{Sa}=.046 ; \mathrm{b}=.109, \mathrm{Sb}=.045)$; Betroffenheit $\rightarrow$ Mediennutzung $\rightarrow$ Partizipation $=$. $012 *(\mathrm{a}=.109, \mathrm{Sa}=.033 ; \mathrm{b}=.109, \mathrm{Sb}=.045)$; Involvierung $\rightarrow$ Mediennutzung $\rightarrow$ Partizipation $=$. $012 *(\mathrm{a}=.106, \mathrm{Sa}=.040 ; \mathrm{b}=.109, \mathrm{Sb}=.045)$; Demokratiezufriedenheit $\rightarrow$ Mediennutzung $\rightarrow$ Partizipation $=.008 *(\mathrm{a}=.075, \mathrm{Sa}=.036 ; \mathrm{b}=.109, \mathrm{Sb}=.045)$. 
bieten direktdemokratische Abstimmungen immer noch die Möglichkeit, dass zusätzliche Informationen hinzugezogen werden, was ja hier auch für die stark Betroffenen und häufigen Tageszeitungsleser der Fall ist. Darüber hinaus ist der Anteil der Hochgebildeten im Sample relativ groß. Dies könnte ebenfalls für die geringen Kommunikationseinflüsse mitverantwortlich sein, da gut informierte Personen mehr eigenes Wissen den medialen Informationen entgegenzusetzen haben (Zaller, 1992).

Unklar bleibt, wie die Medieninhalte zu den hier untersuchten Entscheidungen aussahen und inwiefern es Konsonanz in der Berichterstattung gab. Die Wahrnehmung der Rezipienten lässt jedoch darauf schließen, dass es keine starken Unterschiede in der Berichterstattung gab, sodass allfällige Effekte durch Konsonanz und Vielfalt zumindest auf beiden föderalen Ebenen vermutlich ähnlich ausgefallen wären.

Demokratietheoretisch als weniger wünschenswert für Volksentscheide auf Bundesebene ist einzuschätzen, dass die Meinung zu bundespolitischen Themen nicht so stark auf systematischer Informationsverarbeitung basiert. Andererseits lassen sich die Rezipienten bei der Meinungsbildung zu lokalen Themen stärker von der eigenen Betroffenheit leiten als bei nationalen Themen. Das Fehlen dieses Zusammenhangs bei bundespolitischen Entscheidungen spricht für eine objektivere und weniger eigeninteressengeleitete Meinungsbildung.

Bezüglich der Frage, ob die Bürger kompetent genug für direktdemokratische $\mathrm{Ab}$ stimmungen sind, zeigt sich auf Bundesebene, dass Mediennutzung und persönliche Gespräche die Kompetenz der Befragten bei bundespolitischen Entscheidungen weniger verbessern als bei lokalen Entscheidungen. Weitere Analysen haben gezeigt, dass es vor allem die schon politisch interessierten und betroffenen Rezipienten sind, die die medialen Informationen bei nationalen Entscheidungen nutzen. Gerade bei der Vermittlung von bundespolitischen Themen sollte also das Ziel sein, auch weniger Interessierte anzusprechen. Im Lokalen kann dagegen die Tageszeitungsnutzung zu einer eher kompetenten Abstimmung führen, wobei dies auf nationaler Ebene durch die Orientierung an den Positionen der Parteien potenziell ausgeglichen werden kann.

Zur Frage nach der Beteiligung an Abstimmungen lässt sich resümieren, dass die Nutzung der Medien die Bürger zur Beteiligung an bundesweiten Volksentscheiden mobilisieren kann. Auf beiden föderalen Ebenen sorgt der Einfluss von themenspezifischer Kompetenz dafür, dass die Abstimmung mehrheitlich von einer informierten Stimmbürgerschaft durchgeführt wird. Dies kann auch als problematisch gewertet werden, da durch den Einfluss themenspezifischer Kompetenz gleichzeitig die Selektivität der Partizipation verstärkt wird. Es ist aber zu konstatieren, dass sich diese Problematik bei lokalpolitischen Entscheidungen ebenso stellt wie bei bundespolitischen Entscheidungen.

Die Studie lässt den Schluss zu, dass bei direktdemokratischen Entscheidungen weniger das Augenmerk auf die Differenzierung zwischen den föderalen Ebenen gelegt werden sollte. Da gezeigt werden konnte, dass themenspezifische Aspekte, wie zum Beispiel spezifische Voreinstellungen, für die Meinungsbildung relevanter erscheinen, könnte eine systematische Analyse der Unterschiede je nach Thema weiter Aufschluss über die Meinungsbildungsprozesse bei direktdemokratischen Entscheidungen bringen.

\section{Literatur}

Bachl, M., \& Vögele, C. (2013). „Ich habe die Möglichkeiten in diesem großartigen Land bekommen durch eine tolle Bildung". Inhalte, Wahrnehmung und Wirkungen des bildungspolitischen Debattenteils im TV-Duell vor der Landtagswahl 2011 in Baden-Württemberg. Studies in Communication | Media, 2(3), 367-395. 
Baugut, P., \& Reinemann, C. (2013). Bilanz und Ausblick: Die Analyse politischer Kommunikationskulturen als ein Weg aus der Krise der lokalen Kommunikationsforschung? Studies in Communication | Media, 2(3), 401-429.

Becker, L. B., \& Whitney, D. C. (1980). Effects of Media Dependencies: Audience Assessment of Government. Communication Research, 7 (1), 95-120.

Bonfadelli, H., \& Friemel, T. N. (2012). Learning and Knowledge in Political Campaigns. In H. Kriesi (Ed.), Political Communication in Direct Democratic Campaigns. Enlightening or Manipulating? Basingstoke: Palgrave Macmillan, 169-187.

Bowler, S., \& Donovan, T. (1998): Demanding Choices. Opinion, Voting and Direct Democracy. Ann Arbor: The University of Michigan Press.

de Vreese, C. H. (2007). The Dynamics of Referendum Campaigns. An International Perspective. New York: Palgrave Macmillan.

de Vreese, C. H., \& Semetko, H. A. (2004). News Matters: Influences on the Vote in the Danish 2000 Euro Referendum Campaign. European Journal of Political Research, 43, 699-722.

Eagly, A. H., \& Chaiken, S. (1993). The Psychology of Attitudes. New York: Harcourt Brace Jovanovich.

Eveland, W. P. J. (2001). The Cognitive Mediation Model of Learning from the News: Evidence from Nonelection, Off-Year Election, and Presidential Election Contexts. Communication Research, 28(5), 571-601.

Eveland, W. P. J., Hayes, A. F., Shah, D. V., \& Kwak, N. (2005). Understanding the Relationship Between Communication and Political Knowledge: A Model Comparison Approach Using Panel Data. Political Communication, 22(4), 423-446.

Filla, J., \& Johnson, M. (2010). Local News Outlets and Political Participation. Urban Affairs Review, 45(5), 679-692.

Gabriel, O. W., \& Brettschneider, F. (1997). Werte, Ideologien und Politikpräferenzen. In O. W. Gabriel, F. Brettschneider \& A. Vetter (Hrsg.), Politische Kultur und Wahlverhalten in einer Großstadt. Opladen: Westdeutscher Verlag, 43-68.

Hartleb, F., \& Jesse, E. (2005). Direkte Demokratie in der Bundesrepublik Deutschland: Positionen und Kontroversen. In P. Massing (Hrsg.), Direkte Demokratie. Eine Einführung. Schwalbach: Wochenschau Verlag, 13-38.

Hasebrink, U., \& Schmidt, J.-H. (2012). Informationsrepertoires der deutschen Bevölkerung. Hamburg: Verlag Hans-Bredow-Institut, http://www.hans-bredow-institut.de/webfm_send/ 657 [16.12.2014].

Hefner, D. (2012). Alltagsgespräche über Nachrichten. Medienrezeption, politische Expertise und die wissensbildende Qualität von Anschlusskommunikation. Baden-Baden: Nomos.

Hobolt, S. B. (2007). Taking Cues on Europe? Voter Competence and Party Endorsements in Referendums on European Integration. European Journal of Political Research, 46, 151-182.

Iyengar, S., \& Simon, A. F. (2000). New Perspectives and Evidence on Political Communication and Campaign Effects. Annual Review of Psychology, 51, 149-169.

Kaden, W. (2010). Zu viel Volk schadet Deutschland. Spiegel Online, 04.11.2010, http://www.spie gel.de/wirtschaft/soziales/0,1518,726661,00.html [16.12.2014].

Kim, J., Wyatt, R. O., \& Katz, E. (1999). News, Talk, Opinion, Participation: The Part Played by Conversation in Deliberative Democracy. Political Communication, 16(4), 361-385.

Koch, T., Baden, C., Klötzer, H., \& Müller, E. (2013). Bedingt professionell. Eine Untersuchung zur Professionalität der Kommunikations- und Medienarbeit bayerischer Kommunalpolitiker. Studies in Communication | Media, 2(3), 275-295.

Kost, A. (2006). Bürgerbegehren und Bürgerentscheid in Deutschland. Aus Politik und Zeitgeschichte 10/2006, 25-31.

Kriesi, H. (2005). Direct Democratic Choice: The Swiss Experience. Lanham, Md u. a.: Lexington Books.

Kriesi, H. (2007). The Participation in Swiss Direct-Democratic Votes. In C. H. de Vreese (Hrsg.), The Dynamics of Referendum Campaigns. An International Perspective. New York: Palgrave Macmillan, 117-141. 
Kriesi, H. (2012). The Role of Predispositions. In H. Kriesi (Hrsg.), Political Communication in Direct Democratic Campaigns. Enlightening or Manipulating? Basingstoke: Palgrave Macmillan, 143-167.

Lang, S. (2003). Lokale politische Kommunikation: Öffentlichkeit im Spannungsfeld nationaler und globaler Entwicklungen. In F. Esser \& B. Pfetsch (Hrsg.), Politische Kommunikation im internationalen Vergleich. Grundlagen, Anwendungen, Perspektiven. Wiesbaden: Westdeutscher Verlag, 179-207.

Lupia, A. (1994). Shortcuts versus Encyclopedias: Information and Voting Behaviour in California Insurance Reform Elections. American Political Science Review, 88(1), 63-76.

Maier, J. (2009). Was die Bürger über Politik (nicht) wissen - und was die Massenmedien damit zu tun haben - ein Forschungsüberblick. In F. Marcinkowski \& B. Pfetsch (Hrsg.), Politik in der Mediendemokratie. Wiesbaden: VS Verlag für Sozialwissenschaften, 393-414.

Marcinkowski, F. (2001). Die Lokalpresse im Kontext kommunaler Wahlkämpfe. Eine Fallstudie aus Nordrhein-Westfalen. In H. Abromeit, J.-U. Nieland \& T. Schierl (Hrsg.), Politik, Medien, Technik. Wiesbaden: Westdeutscher Verlag, 260-277.

Marcinkowski, F., \& Marxer, W. (2011). Politische Kommunikation und Volksentscheid. Eine Fallstudie zur Verfassungsreform in Liechtenstein. Baden-Baden: Nomos.

McLeod, J. M., Scheufele, D. A., \& Moy, P. (1999). Community, Communication, and Participation: The Role of Mass Media and Interpersonal Discussion in Local Political Participation. Political Communication, 16 (3), 315-336.

Mehr Demokratie Nordrhein-Westfalen. (o. J.). Die Abstimmungshürde - Hohe Hürde zum Erfolg, http://nrw.mehr-demokratie.de/quorum.html [16.12.2014].

Mende, A., Oehmichen, E., \& Schröter, C. (2012). Medienübergreifende Informationsnutzung und Informationsrepertoires. Media Perspektiven 1/2012, 2-17.

Metag, J. (2013). Lokal kompetenter? Einflüsse von Kommunikations- und Informationsverarbeitungsprozessen auf die Kompetenz bei lokal- und bundespolitischen Entscheidungen. SCM Studies in Communication | Media 2(3), 335-365.

Metag, J. \& Marcinkowski, F. (2012). Strategic, Structural, and Individual Determinants of Online Campaigning in German Elections. Policy \& Internet, 4(3-4), 136-158.

Moy, P., McCluskey, M. R., Kelley, M., \& Spratt, M. A. (2004). Political Correlates of Local News Media Use. Journal of Communication, 54(3), 532-546.

Neller, K. (1999). Lokale Kommunikation: Politikberichterstattung in Tageszeitungen. Wiesbaden: DUV Dt. Univ.-Verl. u. a.

Nicodemus, D. M. (2004). Mobilizing Information: Local News and the Formation of a Viable Political Community. Political Communication, 21(2), 161-176.

Oehmichen, E., \& Schröter, C. (2011). Internet zwischen Globalität und Regionalität. Media Perspektiven, 4/2011, 182-194.

Paletz, D. L., Reichert, P., \& McIntyre, B. (1971). How the Media Support Local Governmental Authority. Public Opinion Quarterly, 35(1), 80-92.

Palmgreen, P., \& Clarke, P. (1977). Agenda-Setting with Local and National Issues. Communication Research, 4(4), 435-452.

Reinemann, C., Fawzi, N., \& Röder, P. (2012). Mehr Beteiligung = bessere Berichterstattung? Ein Vergleich der Presseberichterstattung über die parlamentarische Entscheidung und den Volksentscheid zum Nichtraucherschutzgesetz in Bayern. Studies in Communication | Media, 1(34), 351-380.

Roßteuscher, S., \& Scherer, P. (2013). Wertorientierungen. In J. W. van Deth \& M. Tausendpfund (Hrsg.), Politik im Kontext: Ist alle Politik lokale Politik? Wiesbaden: Springer VS, 67-91.

Schemer, C., Wirth, W., \& Matthes, J. (2008). Out of the Lab into the Field - Zur Operationalisierung und Validierung von Informationsverarbeitungsprozessen in kommunikationswissenschaftlichen Befragungsstudien. In J. Matthes, W. Wirth, G. Daschmann \& A. Fahr (Hrsg.), Die Brücke zwischen Theorie und Empirie: Operationalisierung, Messung und Validierung in der Kommunikationswissenschaft. Köln: von Halem, 28-47.

Scheufele, D. A., Shanahan, J., \& Kim, S.-H. (2002). Who Cares about Local Politics? Media Influences on Local Political Involvement, Issue Awareness, and Attitude Strength. Journalism \& Mass Communication Quarterly, 79(2), 427-444. 
Scheufele, D. A., Nisbet, M. C., Brossard, D., \& Nisbet, E. C. (2004). Social Structure and Citizenship: Examining the Impacts of Social Setting, Network Heterogeneity, and Informational Variables on Political Participation. Political Communication, 21, 315-338.

Schiller, T., \& Mittendorf, V. (2002). Direkte Demokratie. Forschung und Perspektiven. Wiesbaden: Westdeutscher Verlag.

Schmitt-Beck, R. (2000). Politische Kommunikation und Wählerverhalten: Ein internationaler Vergleich. Wiesbaden: Westdeutscher Verlag.

Schnell, R., Hill, P. B., \& Esser, E. (2008). Methoden der empirischen Sozialforschung. München: Oldenbourg.

Schuck, A. R. T., \& de Vreese, C. H. (2009). Reversed Mobilization in Referendum Campaigns: How Positive News Framing Can Mobilize the Skeptics. International Journal of Press/Politics, 14(1), 40-66.

Schuster, J. (2011). Illusion Volksentscheid. Die Welt Kompakt, 10.10.2011. http://www.spiegel.de/ wirtschaft/soziales/debatte-ueber-grossprojekte-zu-viel-volk-schadet-deutschlanda-726661.html [16.12.2014].

Shaker, L. (2009). Citizens' Local Political Knowledge and the Role of Media Access. Journalism \& Mass Communication Quarterly, 86 (4), 809-826.

Sommer, D. (2010). Nachrichten im Gespräch. Wesen und Wirkung von Anschlusskommunikation über Fernsehnachrichten. Baden-Baden: Nomos.

Tenscher, J. (2013). Politische Kommunikation im Kleinen - eine Einleitung. Studies in Communication | Media, 2(3), 265-273.

Tenscher, J., Seeber, G., \& Hallermeyer, M. (2013). Politische Mediennutzung. In J. W. van Deth \& M. Tausendpfund (Hrsg.), Politik im Kontext: Ist alle Politik lokale Politik? Wiesbaden: Springer VS, 191-214.

van Deth, J. W., \& Tausendpfund, M. (2013). Politik im Kontext: Ist alle Politik lokale Politik? Wiesbaden: Springer VS.

Verba, S., Schlozman, K. L., \& Brady, H. E. (1995). Voice and Equality. Civic Voluntarism in American Politics. Cambridge: Harvard University Press.

Verhulst, J., \& Nijeboer, A. (2007). Direkte Demokratie. Fakten, Argumente, Erfahrungen. Brüssel: Democracy international.

Vetter, A. (2011). Lokale Politik als Rettungsanker der Demokratie. Aus Politik und Zeitgeschichte $7-8 / 2011,25-32$.

Winter, C. (1997). Sozialstruktur, Werte, Ideologien und Wahlverhalten. In O. W. Gabriel, F. Brettschneider \& A. Vetter (Hrsg.), Politische Kultur und Wahlverhalten in einer Großstadt. Opladen: Westdeutscher Verlag, 119-146.

Zaller, J. R. (1992). The Nature and Origins of Mass Opinion. Cambridge: Cambridge University Press. 


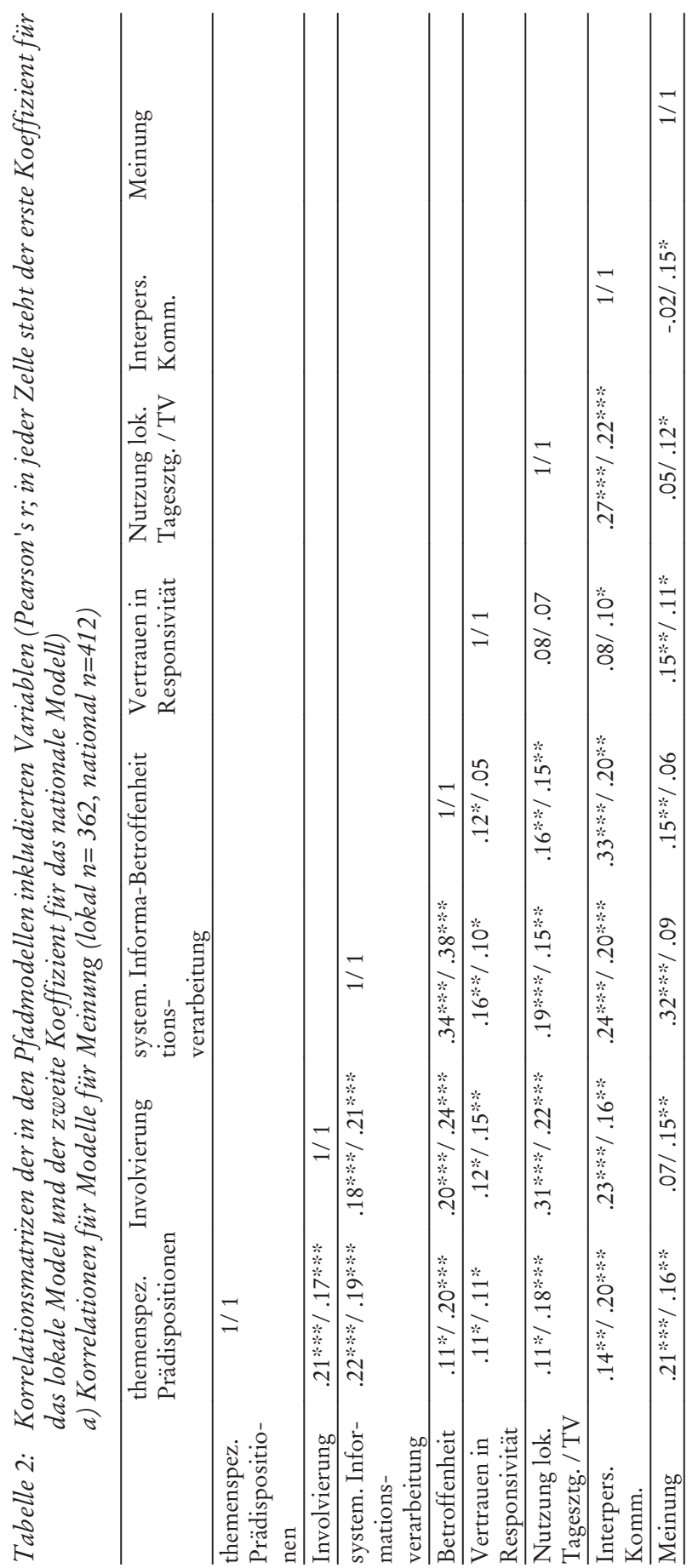




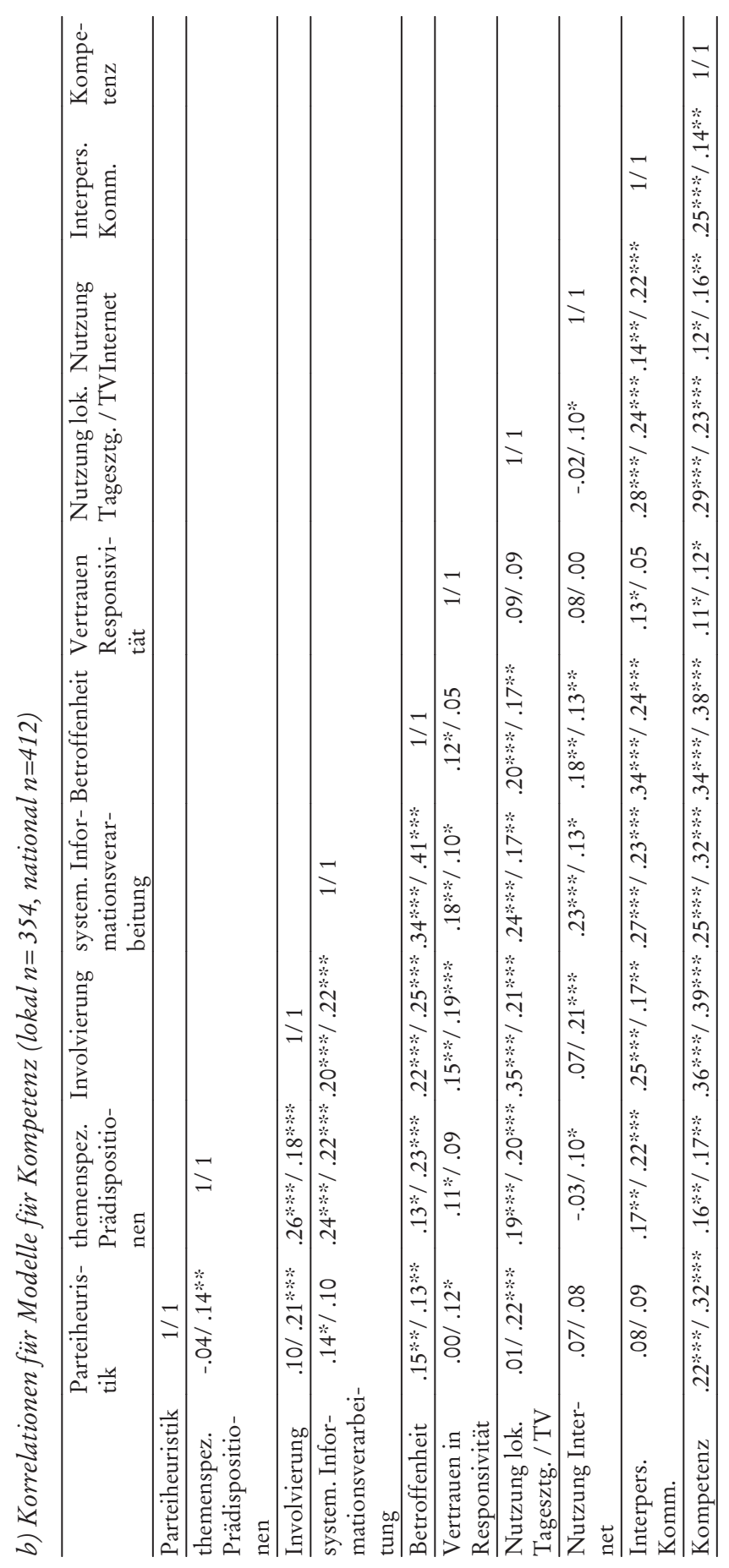




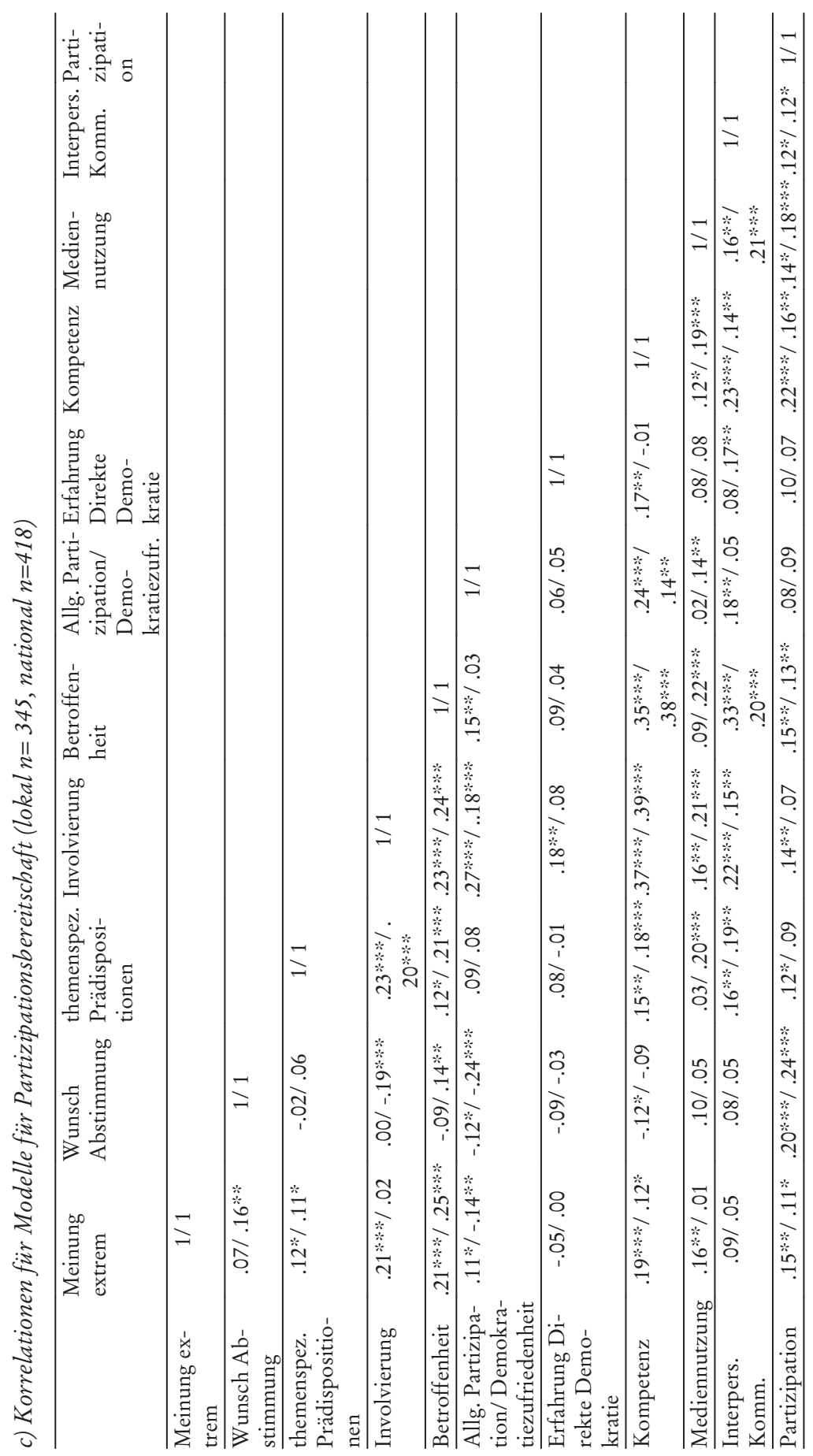

